\title{
CD2AP in mouse and human podocytes controls a proteolytic program that regulates cytoskeletal structure and cellular survival
}

\author{
Suma Yaddanapudi, ${ }^{1}$ Mehmet M. Altintas, ${ }^{2}$ Andreas D. Kistler, ${ }^{2}$ Isabel Fernandez, ${ }^{2}$ \\ Clemens C. Möller, ${ }^{1}$ Changli Wei, ${ }^{2}$ Vasil Peev, ${ }^{2}$ Jan B. Flesche, ${ }^{1}$ Anna-Lena Forst, ${ }^{2}$ Jing Li, ${ }^{2}$ \\ Jaakko Patrakka, ${ }^{3,4}$ Zhijie Xiao, ${ }^{3}$ Florian Grahammer, ${ }^{5}$ Mario Schiffer, ${ }^{6}$ Tobias Lohmüller, ${ }^{7}$ \\ Thomas Reinheckel,7,8 Changkyu Gu, ${ }^{7}$ Tobias B. Huber, 5,8 Wenjun Ju, 9 Markus Bitzer, ${ }^{9}$ \\ Maria P. Rastaldi, ${ }^{10}$ Phillip Ruiz, ${ }^{11}$ Karl Tryggvason, ${ }^{3}$ Andrey S. Shaw, ${ }^{12}$ Christian Faul, ${ }^{2}$ \\ Sanja Sever, ${ }^{1}$ and Jochen Reiser ${ }^{2}$
}

\begin{abstract}
${ }^{1}$ Nephrology Division, Department of Medicine, Harvard Medical School and Massachusetts General Hospital, Charlestown, Massachusetts, USA. 2Division of Nephrology and Hypertension, Leonard Miller School of Medicine, University of Miami, Miami, Florida, USA. ${ }^{3}$ Division of Matrix Biology, Department of Medical Biochemistry and Biophysics, Karolinska Institute, Stockholm, Sweden. ${ }^{4}$ Department of Renal Medicine, Karolinska University Hospital, Stockholm, Sweden. ${ }^{5}$ Renal Division, University Hospital Freiburg, Freiburg, Germany. ${ }^{6}$ Division of Nephrology, Department of Medicine, Hannover Medical School, Hannover, Germany. 7 Institute for Molecular Medicine and Cell Research, Albert-Ludwigs-Universität Freiburg, Freiburg, Germany. ${ }^{8}$ Centre for Biological Signalling Studies (bioss), Albert-Ludwigs-Universität Freiburg, Freiburg, Germany. ${ }^{9}$ Division of Nephrology, Department of Medicine, University of Michigan, Ann Arbor, Michigan, USA. ${ }^{10}$ Renal Research Laboratory, Fondazione IRCCS Ca' Granda Ospedale Maggiore Policlinico and Fondazione D'Amico per la Ricerca sulle Malattie Renali, Milan, Italy. ${ }^{11}$ Department of Pathology and Surgery, Leonard Miller School of Medicine, University of Miami, Miami, Florida, USA. ${ }^{12}$ Howard Hughes Medical Institute and Department of Pathology and Immunology, Washington University School of Medicine, St. Louis, Missouri, USA.
\end{abstract}

Kidney podocytes are highly differentiated epithelial cells that form interdigitating foot processes with bridging slit diaphragms (SDs) that regulate renal ultrafiltration. Podocyte injury results in proteinuric kidney disease, and genetic deletion of SD-associated CD2-associated protein (CD2AP) leads to progressive renal failure in mice and humans. Here, we have shown that CD2AP regulates the TGF- $\beta 1$-dependent translocation of dendrin from the SD to the nucleus. Nuclear dendrin acted as a transcription factor to promote expression of cytosolic cathepsin L (CatL). CatL proteolyzed the regulatory GTPase dynamin and the actin-associated adapter synaptopodin, leading to a reorganization of the podocyte microfilament system and consequent proteinuria. CD2AP itself was proteolyzed by CatL, promoting sustained expression of the protease during podocyte injury, and in turn increasing the apoptotic susceptibility of podocytes to TGF- $\beta 1$. Our study identifies CD2AP as the gatekeeper of the podocyte TGF- $\beta$ response through its regulation of CatL expression and defines a molecular mechanism underlying proteinuric kidney disease.

\section{Introduction}

Several hundred million people worldwide - about 1 in 15 adults - have some form of kidney damage, and every year, millions die prematurely of cardiovascular or renal complications linked to chronic kidney disease (CKD). CKD often begins with urinary protein loss (proteinuria), an early sign of kidney injury that constitutes a risk factor for further progressive destruction of the kidney, a process that can last from weeks to several years (1). Proteinuria stems from injury to podocytes, terminally differentiated cells that reside in the kidney glomeruli, the location of the renal filtration barrier. The function of podocytes is primarily based on their intricate structure, which consists of a cell body, major processes, and interdigitating foot processes (FPs), which are actin-driven membrane extensions. At the interface of adjacent FPs, a specialized intercellular junction known as the slit diaphragm (SD) is formed. Nephrin, a key structural and signaling transmembrane protein of

Authorship note: Suma Yaddanapudi and Mehmet M. Altintas contributed equally to this work.

Conflict of interest: Sanja Sever and Jochen Reiser have issued and filed US patents concerning methods and technologies related to the diagnosis, mechanisms, and treatment of proteinuric kidney disease and stand to gain royalties from their future commercialization

Citation for this article: J Clin Invest. 2011;121(10):3965-3980. doi:10.1172/JCI58552. the $\mathrm{SD}$, recruits proteins such as podocin, $\mathrm{CD} 2 \mathrm{AP}$, and Nck to the podocyte membrane (2). One of the earliest events in the development of podocyte dysfunction is the disruption of FPs (referred to as FP effacement), which causes proteinuria, the first clinical sign in CKD. Once podocytes are injured, there are 2 possible outcomes: (a) proteinuria resolves and podocyte structure normalizes, or (b) renal function declines, resulting in progressive glomerular and consecutive tubular destruction (3). The latter outcome is characterized by an increased occurrence of podocyte apoptosis, a recognized event commonly observed during renal disease progression (4). Generally, the reason for heightened susceptibility of podocytes during proteinuria is a phenomenon that is not well understood.

Recent years have shed light on the molecular makeup of the SD and podocyte FPs, mainly through human and mouse genetic studies (5). Clinically, acquired forms of glomerular dysfunction, such as those seen in diabetes mellitus, are far more common and follow similar patterns of injury. A key event in the development of podocyte FP effacement and proteinuria lies in the induction of a cytosolic form of the protease cathepsin L (CatL; encoded by Ctsl) in podocytes that has recently been characterized in triggering the disease process $(6,7)$. Ctsl mRNA is found in all tissues, but was characterized as an enriched glomerular-specific transcript compared with other segments of the kidney (8). Via mechanisms of 

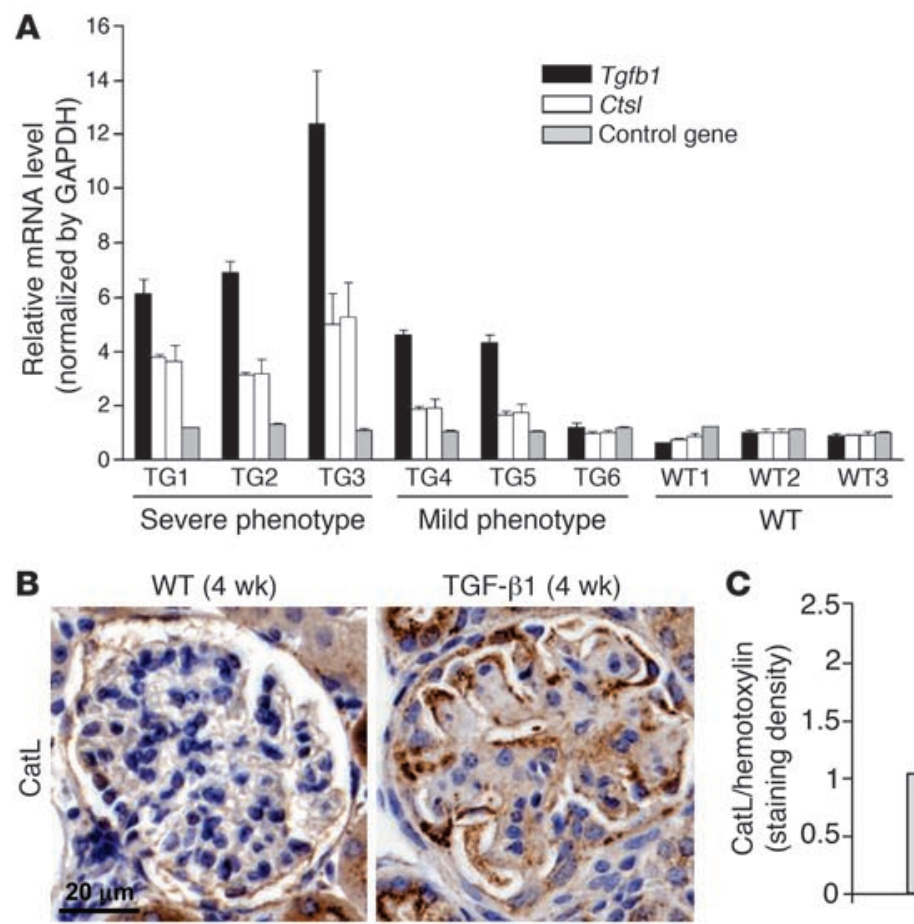

D
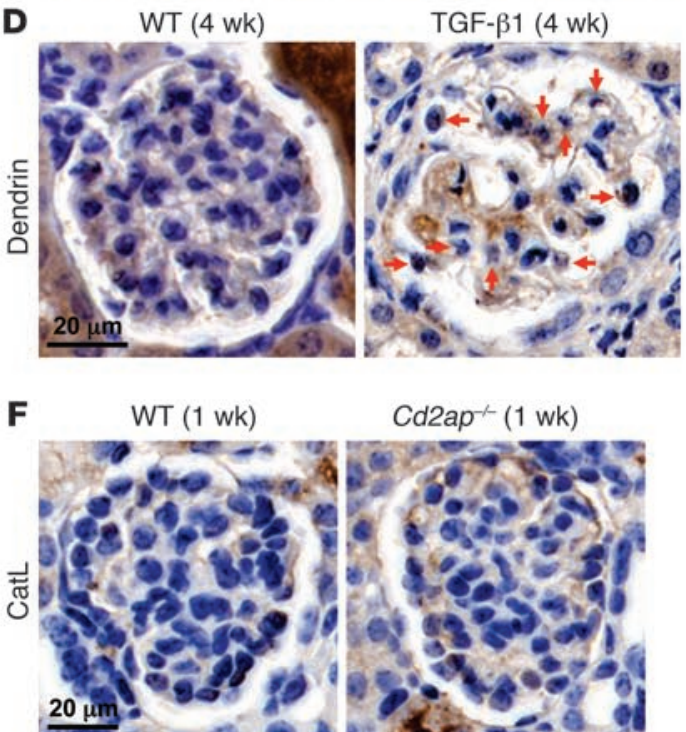

$\mathrm{Cd}_{2 \mathrm{ap}}^{-/}(1 \mathrm{wk})$

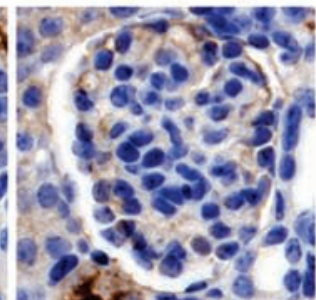

G

$$
\text { WT (3 wk) }
$$

$\mathrm{Cd}_{2 a p^{-/}}(3 \mathrm{wk})$

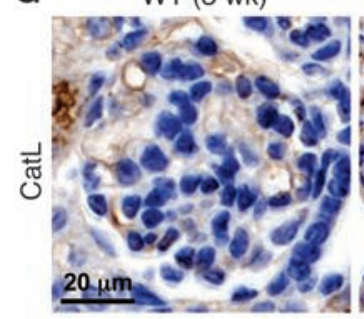

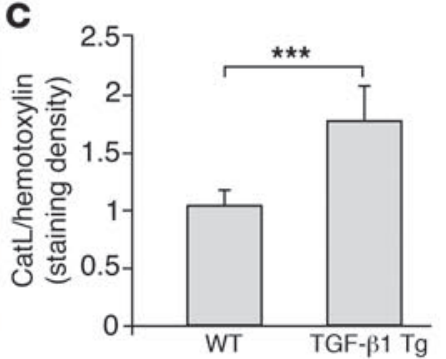

E

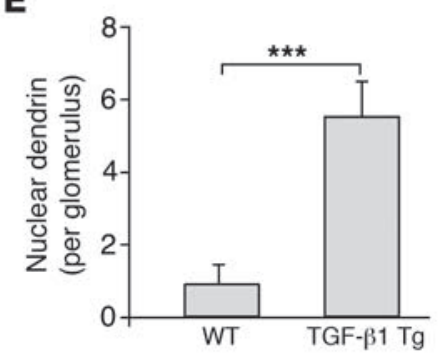

Figure 1

High level of TGF- $\beta 1$ induces expression of CatL. (A) Levels of Tgfb1, Ctsl, and control gene in glomeruli of TGF- $\beta 1 \mathrm{Tg}$ and WT animals. Phenotype categorization is as described previously (23). (B) H\&Estained sections of the renal cortex, showing increased CatL staining in TGF- $\beta 1 \mathrm{Tg}$ mice. Kidneys of WT and TGF- $\beta 1 \mathrm{Tg}$ mice were stained for CatL using anti-cytosolic CatL antibody, and nuclei were stained using DAPI. (C) CatL staining levels in sections from $\mathbf{B}$. ${ }^{* * *} P<0.001$. (D) H\&E-stained sections showing dendrin localization in the nucleus of the podocytes in TGF- $\beta 1 \mathrm{Tg}$ animals (arrows). Kidneys of WT and TGF- $\beta 1$ mice were stained for dendrin using antidendrin antibody. (E) Dendrin staining levels in sections from $\mathbf{D}$. ${ }^{* *} P<0.001$. (F and $\mathbf{G}$ ) H\&E-stained sections showing increased CatL staining in glomerulus of 3-week-old Cd2ap-/- mice (arrows). Kidneys of WT and $\mathrm{Cd}_{2} \mathrm{ap}^{-/-}$mice were stained for CatL using anti-cytosolic CatL antibody, and nuclei were stained using DAPI. (H) Dendrin localization in glomerulus of WT and Cd2ap ${ }^{-/-}$mice at different stages of development. WT1 was used to stain podocyte nuclei (red). In the young glomerulus, dendrin (green) was localized at the membrane (ribbon staining pattern), without any colocalization with WT1. At 4 weeks, dendrin staining was still localized to the plasma membrane in WT mice, but was predominantly found in the nucleus costaining with WT1 in Cd2ap ${ }^{-/-}$mice. Scale bars: $20 \mu \mathrm{m}$ (B, D, F, and G); $30 \mu \mathrm{m}(\mathbf{H})$.
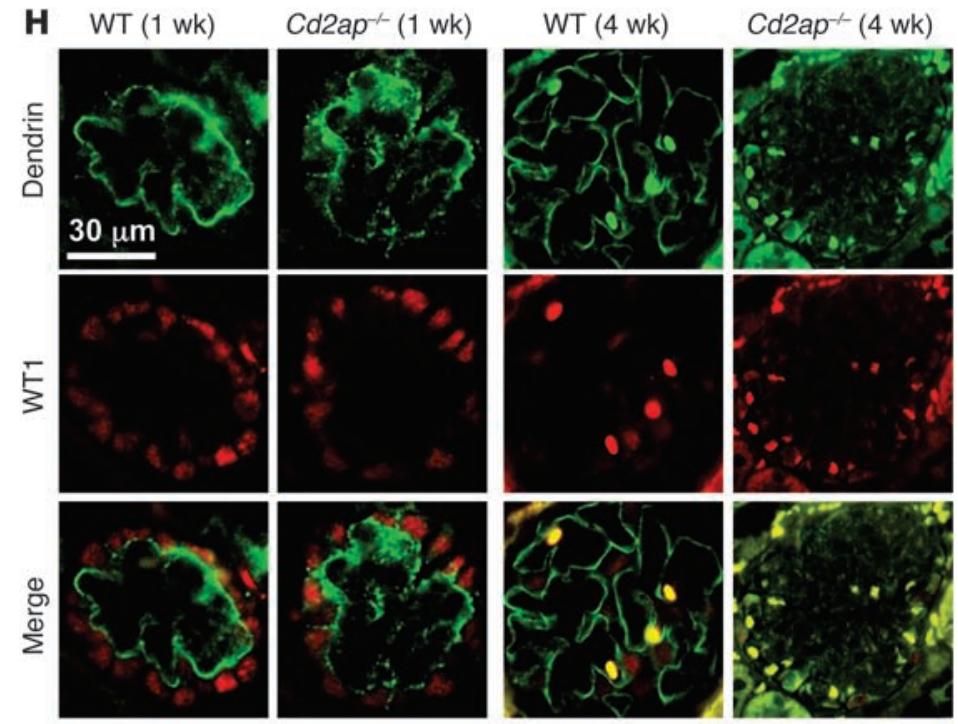

alternative translation of $\mathrm{Ctsl} \mathrm{mRNA}$, a cytosolic CatL protein that lacks the lysosomal targeting sequence can be produced in a few cell types, including podocytes $(6,9)$. Physiological functions of cytosolic CatL include processing of transcription factors (10) as well as processing of histone $\mathrm{H} 3$ during embryonic stem cell differentiation in mice (11). In podocytes, cytosolic CatL proteolyzes the large GTPase dynamin (6) and the actin-binding protein synaptopodin (7). Both events result in disorganization of the podo- 
cyte actin cytoskeleton and FP effacement. FP effacement can be inhibited by blocking CatL activity or by protection of the target proteins from CatL cleavage $(6,7)$.

Thus far, it is unclear how the expression of cytosolic CatL is regulated. An important genetic model to study the sequela of podocyte injury and glomerular disease is the $C d 2 a p^{-/-}$mouse model (12). $\mathrm{CD} 2 \mathrm{AP}$, a scaffolding protein, is essential for proper signaling at the $\operatorname{SD}(13,14)$. Thus, homozygous CD2AP mutation or haploinsufficiency of the human CD2AP gene predisposes to renal disease (15), and mice lacking 1 copy of $C d 2 a p$ develop glomerular renal failure (16). Importantly, $\mathrm{Tg}$ mice expressing CD2AP only in podocytes prevented the development of proteinuria, which demonstrated that the renal failure is solely due to loss of CD2AP in podocytes (17). Despite these findings, the molecular mechanism by which CD2AP regulates function of podocyte has not been fully understood. It has been shown that loss of CD2AP leads to increased expression of TGF- $\beta 1$ in podocytes and apoptosis $(18,19)$, demonstrating that $\mathrm{CD} 2 \mathrm{AP}$ somehow regulates podocyte survival by regulating expression of TGF- $\beta 1$. Furthermore, high levels of TGF- $\beta 1$ have been associated with translocation of dendrin from the SD to the nucleus, which in turn increased podocyte apoptosis (20). Dendrin binds CD2AP and nephrin at the SD, which suggests that loss of $\mathrm{CD} 2 \mathrm{AP}$ and subsequent increase in TGF- $\beta 1$ expression in podocytes might drive dendrin translocation in cells lacking CD2AP. Here, we showed that genetic deletion or proteolytic degradation of CD2AP allowed for an increase in podocyte TGF- $\beta 1$ signaling that in turn drove translocation of dendrin from the SD into the nucleus. We next identified dendrin as a CatL transcription factor specifically promoting expression of cytosolic CatL, a finding we believe to be novel. Cytosolic CatL in turn drove reorganization of the actin cytoskeleton by proteolytically processing dynamin and synaptopodin. Alteration in the actin cytoskeleton rendered podocytes hypersensitive to proapoptotic signals. The prolonged presence of cytosolic CatL led to sustained loss of CD2AP, which in turn promoted dendrin translocation and drove sustained expression of cytosolic CatL, establishing progressive renal disease. In summary, our results demonstrate that CD2AP plays an unexpected role in the regulation of the expression of cytosolic CatL and link SD injury with a proteolytic program that underlies progressive renal disease.

\section{Results}

Increased TGF- $\beta 1$ signaling induces CatL expression. Most forms of glomerular diseases, such as diabetic nephropathy, are associated with increases in TGF- $\beta 1$ signaling (21). We have previously shown that Ctsl mRNA and CatL protein levels are upregulated in glomeruli of patients with diabetic nephropathy (6). Thus, we examined whether mice $\mathrm{Tg}$ for TGF- $\beta 1$ exhibit elevated CatL expression. These animals, which express TGF- $\beta 1$ in the liver controlled by an albumin promoter, display elevated plasma concentrations of TGF- $\beta 1$, exhibit podocyte apoptosis at an early stage of disease, and develop variable degrees of CKD and proteinuria $(22,23)$. Because all animals have high levels of circulatory TGF- $\beta 1$, which in itself is not sufficient to explain the variability in the kidney phenotype (glomerulosclerosis), we used real-time PCR (RT-PCR) to examine mRNA levels of Tgfb1 in isolated kidneys of TGF- $\beta 1 \mathrm{Tg}$ animals. There was an increase in mRNA levels for Tgfb1, which correlated well with severity of the kidney phenotype (Figure 1). A similar correlation was detected with respect to Ctsl mRNA levels in glomeruli. Together, these data showed that there was a strong association between $T g f b 1$ and $C t s l$ mRNA levels and severity of glo- merulosclerosis ( $r=0.96$ and $r=0.97$, respectively). The increase in Ctsl mRNA levels in glomeruli translated into an increase in CatL protein levels in podocytes, as revealed by immunohistochemical staining for CatL (Figure 1, B and C). Together, these data showed that increased exogenous levels of TGF- $\beta 1$ can increase both endogenous kidney TGF- $\beta 1$ levels and CatL expression.

It has previously been shown that high levels of TGF- $\beta 1$ induce translocation of dendrin from the plasma membrane to the nucleus of podocytes in culture (20). In line with these studies, we found increased nuclear dendrin staining in TGF- $\beta 1 \mathrm{Tg}$ mice (Figure 1, D and E). To explore the identified connection between increased TGF- $\beta 1$ and CatL levels in the glomerulus and TGF- $\beta 1-$ dependent nuclear localization of dendrin in podocytes, we turned to the $C d 2 a p^{-/-}$mouse model of progressive kidney disease. $C d 2 a p^{-/-}$ mice are born normal, but develop heavy proteinuria and renal failure approximately 4 weeks after birth and die usually at 6 weeks of age $(12,17)$. As glomerular disease progresses in these mice, podocyte apoptosis increases, driven in part by an increase in intraglomerular TGF- $\beta 1$ signaling (18). Given the increased TGF- $\beta 1$ levels in podocytes lacking CD2AP at the time of proteinuria onset, these mice represent an ideal model to examine the connection between TGF- $\beta 1$ signaling and CatL expression in podocytes. We first examined whether increased CatL levels coincide with proteinuria onset in $C d 2 a p^{-/-}$animals. Whereas glomeruli of both WT and Cd2ap $p^{-/-}$mice exhibited low-level staining for CatL at 1 week, staining for CatL increased in glomeruli of 3-week-old $C d 2 a p^{-/-}$mice (Figure 1, F and G). The staining indicated that a majority of CatL was present within the podocytes (Figure 1G, arrows). Next, we examined whether dendrin could be found in the nucleus in $C d 2 a p^{-1-}$ animals. The classical plasma membrane staining was observed for dendrin in $\mathrm{Cd} 2 \mathrm{ap}^{-/-}$mice at 1 week of age, when mice were still healthy (Figure $1 \mathrm{H}$ ). Importantly, dendrin exhibited a nuclear staining pattern in 4-week-old $C d 2 a p^{-/-}$ mice (Figure $1 \mathrm{H}$ ), which demonstrated that dendrin translocated into the nucleus of podocytes during the early stages of podocyte injury. In contrast, dendrin staining in the glomeruli of 1- or 4-week-old WT mice exhibited a characteristic membrane pattern (Figure $1 \mathrm{H}$ ), in agreement with its SD localization (20). Together, our data extended prior observations by identifying a link among loss of the SD adaptor protein CD2AP, increase in autocrine levels of TGF- $\beta 1$, dendrin translocation into the nucleus, and increased levels of Cat $\mathrm{L}$ in podocytes.

Loss of CD2AP leads to expression of cytosolic CatL in podocytes. To elucidate the molecular mechanism that regulates expression of CatL in a TGF- $\beta 1$-dependent manner, we turned to podocytes in culture. In agreement with the animal model and previously published studies (17), conditionally immortalized podocytes derived from $C d 2 a p^{-/-}$mice were indistinguishable from the WT podocytes, with well-defined focal adhesions (FAs) and stress fibers (Figure 2A, left panels). Similar to the course of pathology in $C d 2 a p^{-/-}$mice, $C d 2 a p^{-/-}$podocytes cultured for more than 6 weeks developed distinct alteration in the actin cytoskeleton, loss of mature FAs and increased number of focal complexes, and loss of well-defined stress fibers and dramatically increased number of transverse arcs (Figure 2A). The alteration in the actin cytoskeleton was similar to that observed in cells treated with LPS $(6,24)$ and was consistent with FP effacement at the onset of proteinuria in $C d 2 \mathrm{ap}^{-/-}$mice $(12,15)$. Because increased glomerular levels of TGF- $\beta 1$ that can act in a paracrine/autocrine fashion has been shown to occur at the onset of proteinuria (18), 


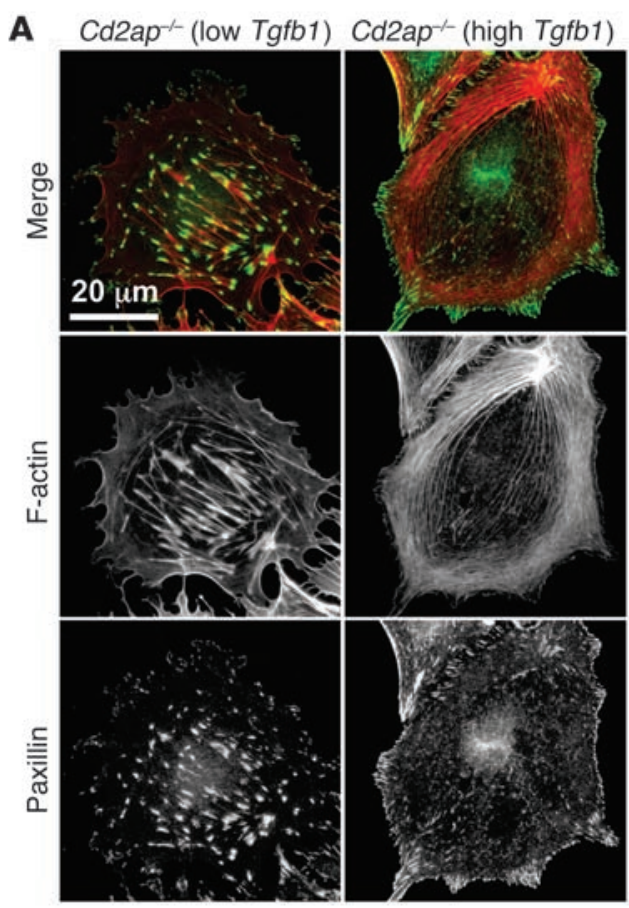

B
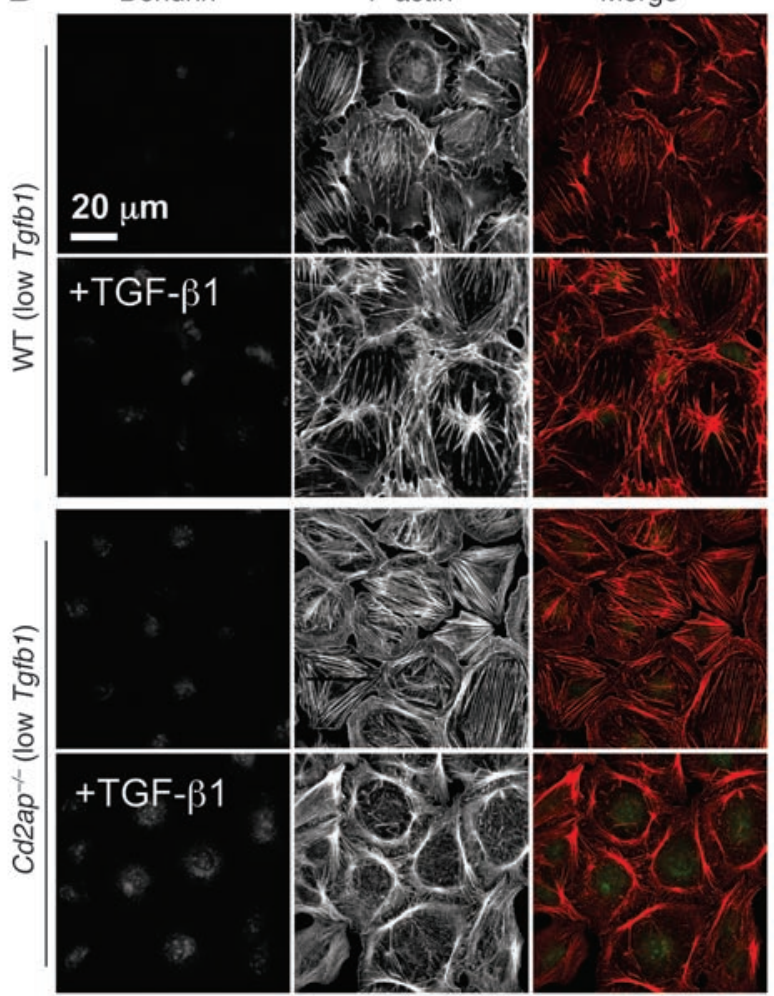
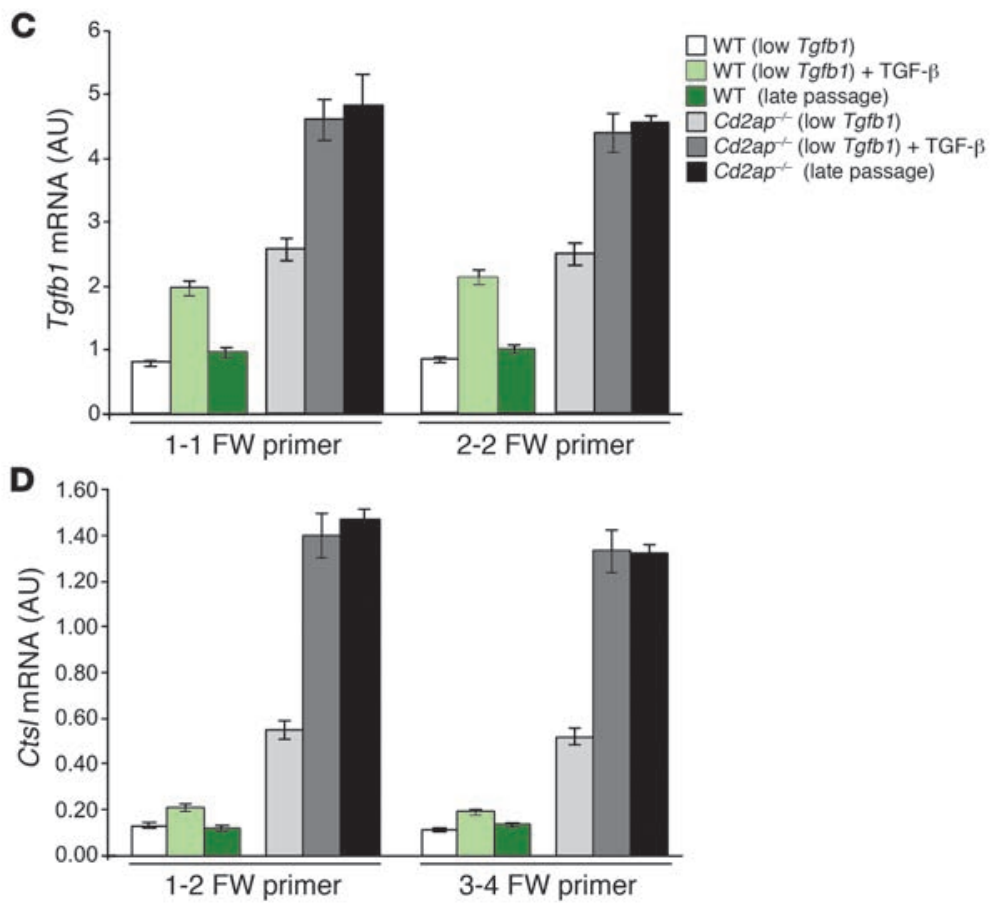

E

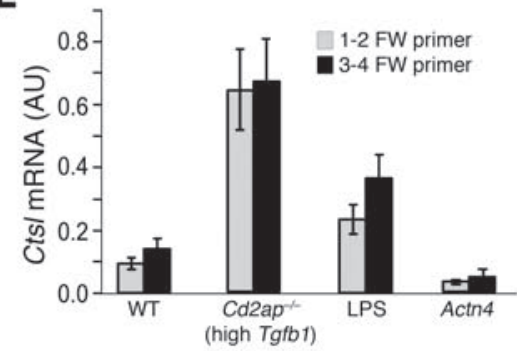

$\mathbf{F}$

$\mathrm{Cd}^{2 a \mathrm{p}^{-1-}}$

Low Tgfb1 High Tgfb1

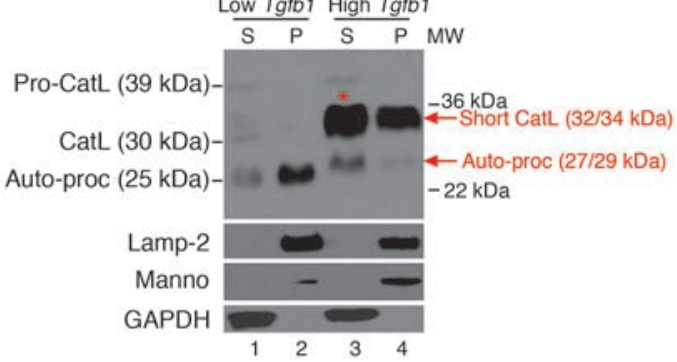




\section{Figure 2}

Prolonged loss of CD2AP leads to expression of cytosolic CatL. (A) Actin cytoskeleton and FA organization in fully differentiated lowand high-Tgfb1 $\mathrm{Cd}_{2} \mathrm{ap}^{-/-}$cells. Note the loss of well-defined stress fibers and dramatic increase in number of transverse arcs in highTgfb1 $\mathrm{Cd}_{2} \mathrm{ap}^{-/-}$cells. FAs and F-actin were visualized with anti-paxillin antibodies and rhodamine-phalloidin, respectively. (B) Low-Tgfb1 $\mathrm{Cd}_{2} \mathrm{ap}^{-/-}$podocytes could be transformed into high-Tgfb1 $\mathrm{Cd} 2 \mathrm{ap} \mathrm{p}^{-/-}$ podocytes by high TGF- $\beta 1$ levels. WT and low-Tgfb1 $\mathrm{Cd}_{2 \mathrm{ap}}^{-/-}$passage cells were treated with $5 \mathrm{ng} / \mathrm{ml}$ TGF- $\beta 1$ in the media for 24 hours. Actin cytoskeleton was monitored by staining cells with rhodaminephalloidin, and dendrin localization was monitored using anti-dendrin antibody (green). (C-E) mRNA levels for Tgfb1 (C) and Ctsl (D and E), determined by RT-PCR in podocytes. When indicated, cells were treated with $5 \mathrm{ng} / \mathrm{ml} \mathrm{TGF}-\beta 1$ for 24 hours or with $50 \mu \mathrm{M}$ LPS for 24 hours. Podocytes expressing Actn 4 mutant are also shown in E. (F) Subcellular fractionation of low- and high-Tgfb1 $\mathrm{Cd} 2 a p^{-/}$podocytes in isotonic sucrose. Total proteins from soluble (S) and particulate (P) fractions were probed for CatL and for the lysosomal protein markers Lamp-2 and mannosidase (Manno), then analyzed by Western blotting. GAPDH was used as a loading control. Strong cytosolic CatL induction (asterisk) was observed in both soluble and pellet fractions of high-Tgfb1 Cd2ap ${ }^{-/-}$podocytes. Scale bars: $20 \mu \mathrm{m}$.

we compared Tgfb1 mRNA levels in $C d 2 a p^{-/-}$podocytes that contained WT organization of the actin cytoskeleton (early passage) and those with altered actin cytoskeleton (late passage). Indeed, late-passage $C d 2 a p^{-1-}$ podocytes exhibited higher Tgfb1 mRNA levels than did early-passage $C d 2 a p^{-/-}$podocytes (Figure $2 \mathrm{C}$ ). To further explore the connection between high Tgfb1 mRNA levels and actin cytoskeleton alterations, we examined whether addition of high levels of recombinant TGF- $\beta 1$ in the media could induce alteration of the podocyte actin cytoskeleton. Culturing of WT podocytes with $5 \mathrm{ng} / \mathrm{ml}$ TGF- $\beta 1$ did not induce alteration in their actin cytoskeleton (Figure 2B). In contrast, identical treatment of early-passage $\mathrm{Cd} 2 \mathrm{ap}^{-/-}$podocytes induced dramatic alteration of their actin cytoskeleton (Figure $2 \mathrm{~B}$ ). This TGF- $\beta 1-$ dependent alteration of the actin cytoskeleton coincided with increased dendrin staining in the nucleus in $\mathrm{Cd} 2 \mathrm{ap}^{-/-}$cells (Figure $2 \mathrm{~B})$. Notably, we detected dendrin in the nucleus to some degree even in WT cells (ref. 20; Figure 2B; and Supplemental Figure 1A; supplemental material available online with this article; doi:10.1172/JCI58552DS1).

Even at the basal state, $C d 2 a p^{-/-}$cells exhibited higher $T g f b 1$ mRNA levels than did WT cells, and this level was further increased upon culturing cells in TGF- $\beta 1$ rich media (Figure $2 \mathrm{C}$ ). Thus, podocytes lacking CD2AP were susceptible to increased levels of TGF- $\beta 1$ in media, which in turn drove reorganization of their actin cytoskeleton. Consequently, Cd2ap ${ }^{-/-}$podocytes with low $\mathrm{Tg} f b 1$ mRNA levels exhibited WT organization of the actin cytoskeleton, and those with high $T g f b 1 \mathrm{mRNA}$ levels exhibited altered organization of the actin cytoskeleton (Figure 2, A and B). While at present we do not understand the molecular mechanism by which CD2AP regulates expression of TGF- $\beta 1$ in podocytes, experimentally, the switch from low to high Tgfb1 was achieved by prolonged culturing of $C d 2 a p^{-1-}$ podocytes to allow for endogenous autocrine TGF- $\beta 1$ signaling (Figure 2A and Supplemental Figure 1A) as well as by adding high levels of recombinant TGF- $\beta 1$ into the media to rapidly transduce the Tgfb1 signal (Figure 2, B and C). In both instances, the levels of endogenous Tgfb1 mRNA correlated with nuclear dendrin staining (Figure 2B and Supplemental Figure 1A).
Importantly, the increase in TGF- $\beta 1$ induced an increase in Ctsl mRNA levels in $C d 2 a p^{-/-}$cells. In contrast, expression of Ctsl mRNA in WT cells was resistant to high levels of TGF- $\beta 1$ (Figure 2D). However, even in WT cells, the higher the TGF- $\beta$ level in the media, the higher the level of Ctsl mRNA (Supplemental Figure 1B), which suggests that prolonged high levels of exogenous TGF- $\beta$ might trigger an increase in Ctsl mRNA. We made similar observations when Ctsl mRNA levels were compared among WT cells, high-Tgfb1 $C d 2 a p^{-/-}$cells, and cells treated with LPS (Figure 2E). Increased Ctsl mRNA was not observed in podocytes expressing a gain-offunction mutation in the $\alpha$-actinin-4 gene (Actn 4 ; Figure 2E) or in podocytes in which dynamin or synaptopodin were downregulated (Supplemental Figure 1C). These data, consistent with our findings in animals (Figure 1, A and B), suggested that increased TGF- $\beta 1$ signaling has a specific effect on CatL expression.

Upregulation of $\mathrm{Ctsl}$ mRNA resulted in a dramatic increase in the amount of cytosolic CatL (also referred to as short-form CatL; Figure 2F, lane 3; Supplemental Figure 1D; and ref. 9). Indeed, cytosolic CatL was also found in the nucleus (Supplemental Figure $1 \mathrm{E}$ ), as described previously (10). We and others have shown that cytosolic CatL downregulates protein levels of dynamin (6), synaptopodin, and RhoA (7). In agreement with these findings, high-Tgfb1 $C d 2 a p^{-/-}$cells exhibited lower levels of dynamin, synaptopodin, and RhoA without affecting the level of $\alpha$-actinin- 4 (Supplemental Figure 1F). The decreased protein levels were not caused by downregulation of mRNA for those proteins (Supplemental Figure 1, G and I), which suggests that decreased levels are caused by proteolytic processing of these proteins by cytosolic CatL. Together, these data suggest that $C d 2 a p^{-/-}$podocytes in culture phenocopy alterations that occur in podocytes in $\mathrm{Cd} 2 \mathrm{ap}^{-/-}$animals at the onset of proteinuria: increased expression of endogenous TGF- $\beta 1(18,19)$, dendrin translocation into the nucleus (Figure $1 \mathrm{H}$ ), and increased expression of CatL (Figure 1G)

Cytosolic CatL alters the actin cytoskeleton in $\mathrm{Cd} 2 \mathrm{a} \mathrm{p}^{-1-}$ cells. Our study identified the presence of cytosolic CatL in high-Tgfb1 Cd2ap ${ }^{-1-}$ podocytes. To further explore the hypothesis that the presence of cytosolic CatL drives alterations of the actin cytoskeleton in $C d 2 a p^{-/-}$cells by proteolytically processing a subset of proteins, we inhibited CatL activity by lentivirus-based shRNAs and by using the small-molecule cysteine protease inhibitor E64, which blocks CatL. Downregulation of CatL by RNAi was confirmed using RT-PCR and Western blot analysis (Figure 3, A and B). 3 different shRNAs downregulated CatL protein levels 70\%-90\% and significantly reduced CatL activity in the cytoplasm of high-Tgfb1 $\mathrm{Cd} 2 \mathrm{ap}^{-/-}$cells (Figure 3, B and C). In agreement with the fact that the enzymatic assay measures activities of both CatL and CatB, addition of CA074, a specific CatB inhibitor, resulted in further lowering of the measured activity to the basal level (Figure 3C), thus defining the specificity of the assay. Therefore, the CatB inhibitor CA074 was included in all subsequent assays. As shown in Figure 3D, downregulation of CatL in high-Tgfb1 Cd2ap ${ }^{-/-}$cells by shRNA-C6 resulted in CatL activity similar to that observed in WT cells. Immunofluorescence and Western blot analysis demonstrated that CatL was active in the cytoplasm of high-Tgfb1 Cd2ap $\mathrm{p}^{-/-}$cells (Figure 2F). If loss of dynamin and synaptopodin was indeed the result of identified activity of cytosolic CatL, then treatment of cells with E64 or downregulation of CatL expression by shRNA should exhibit a protective effect on the loss of these proteins. As expected, both treatments resulted in increased levels of dynamin, synaptopodin, and RhoA (Figure 3, E-G). In agree- 


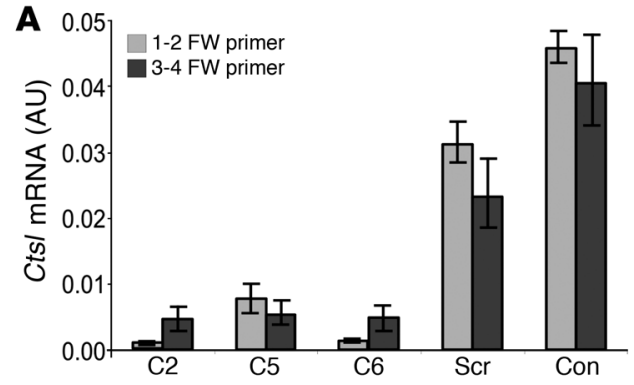

B
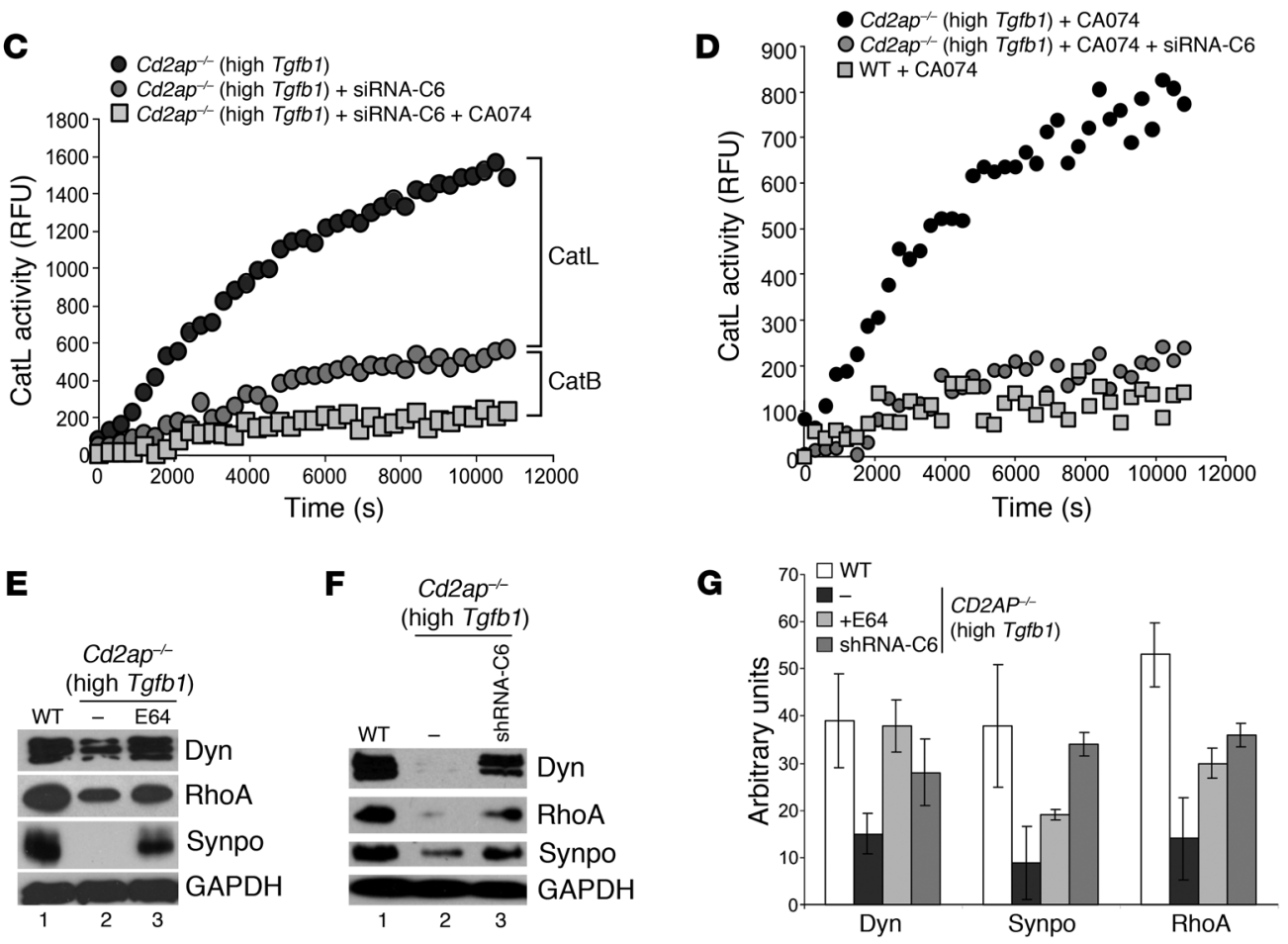

Figure 3

Cytosolic CatL activity regulates actin cytoskeleton in Cd2ap ${ }^{-/-}$cells. (A) Cts/ mRNA levels, determined by RT-PCR, in high-Tgfb1 Cd2ap ${ }^{-/-}$ podocytes infected with different shRNA constructs $(\mathrm{C} 2, \mathrm{C} 5, \mathrm{C} 6)$ downregulating endogenous CatL. Con, high-Tgfb1 Cd2ap ${ }^{-/}$podocytes not infected with lentiviruses; Scr, cells infected with lentiviruses expressing a scrambled oligo. (B) CatL levels in high-Tgfb1 Cd2ap ${ }^{-/-}$podocytes infected with lentiviruses expressing different shRNA constructs to downregulate CatL at relative volumes as indicated. (C) Time course of CatL activity in high-Tgfb1 $\mathrm{Cd} 2 a \mathrm{p}^{-/-}$podocytes and in high-Tgfb1 $\mathrm{Cd} 2 \mathrm{ap} \mathrm{p}^{-/}$podocytes infected with lentiviruses to downregulate CatL in the absence and presence of CA074 at neutral pH. (D) Time course of CatL activity in the presence of CA074 in high-Tgfb1 Cd2app ${ }^{-/-}$podocytes, high-Tgfb1 $\mathrm{Cd}_{2} \mathrm{ap}^{-/-}$podocytes infected with lentiviruses to downregulate CatL, and WT podocytes at neutral pH. (E and F) Protein levels in WT cells, high-

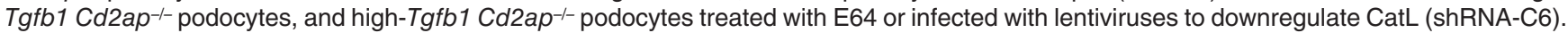
Dyn, dynamin; Synpo, synaptopodin. GAPDH was used as a loading control. (G) Quantitation of protein levels from Western blots in E and F.

ment with increased levels of these proteins in treated high-Tgfb1 $\mathrm{Cd} 2 \mathrm{ap}^{-/-}$cells, CatL inhibition partially restored the number of podocyte FAs as well as stress fibers (Figure 4, A and B). Together, these data demonstrated an increase in cytosolic CatL in high$T g f b 1 \mathrm{Cd} 2 \mathrm{ap}^{-1-}$ podocytes, which in turn drives reorganization of the actin cytoskeleton through proteolytic processing of a subset of proteins that regulates the actin cytoskeleton.

Interestingly, downregulation of CatL in WT podocytes, either by siRNA or by addition of E64 (Supplemental Figure 2, A-D), resulted in an increase in FA size (Supplemental Figure 2, E-G). Thus, although the number of FAs per cell was similar, there was a shift toward more mature FAs. This could be explained by the increase in levels of dynamin, synaptopodin, and - to some extent
- RhoA (Supplemental Figure 2H). Together, these data suggest that endogenous levels of cytosolic CatL regulate turnover of the FAs in cultured podocytes.

Nuclear dendrin regulates expression of cytosolic CatL. Our data identified a link between dendrin localization in the nucleus and expression of cytosolic CatL. To test whether nuclear dendrin (encoded by Ddn) directly induces expression of cytosolic CatL, dendrin was downregulated in high-Tgfb1 $C d 2 a p^{-/-}$cells using shRNAs (Figure $5, A$ and $B$ ). This led to partial restoration of FAs and stress fibers within the cell body - demonstrating that loss of dendrin had a direct consequence on actin organization in high-Tgfb1 $\mathrm{Cd} 2 \mathrm{ap} \mathrm{p}^{-/-}$ cells - that was also indicated by FAs formed within the cell body that connected short actin filaments (Figure 5, C and D). Strikingly, 
A
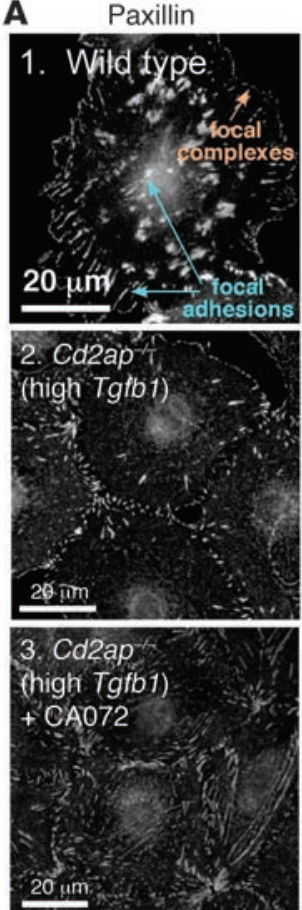

4. Cd2ap
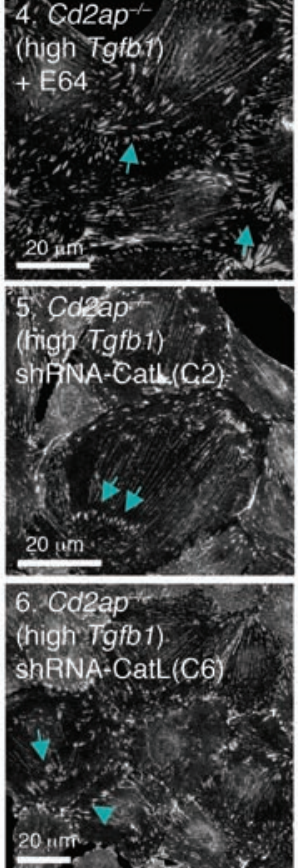

F-actin
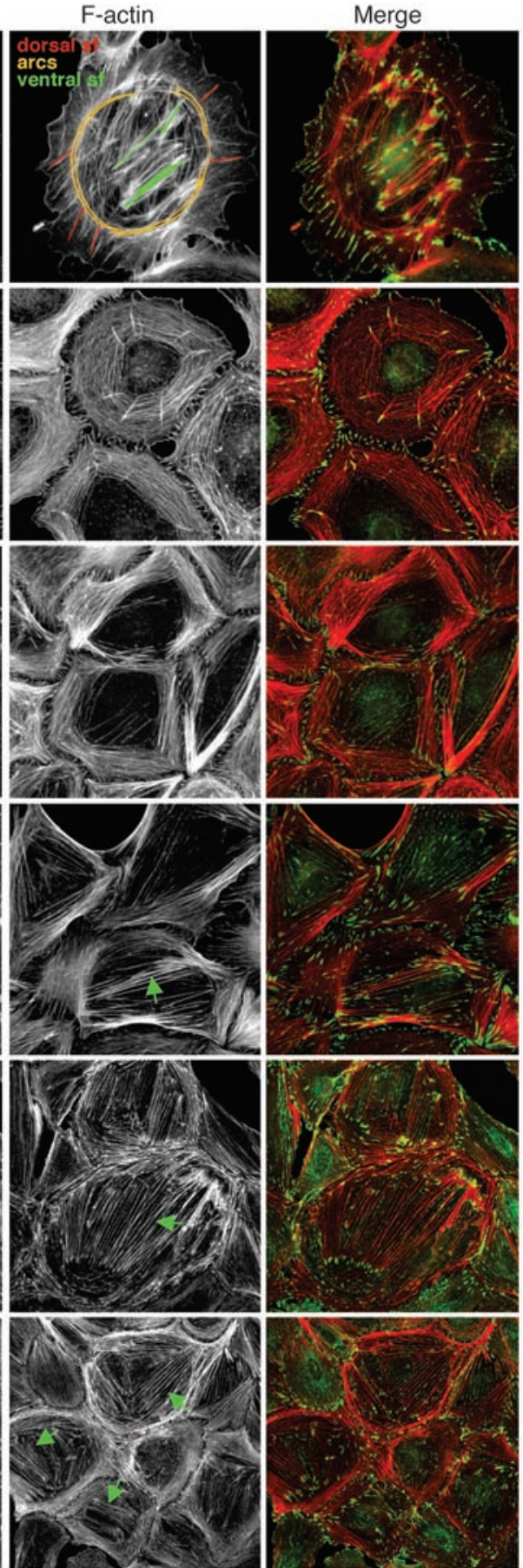

B

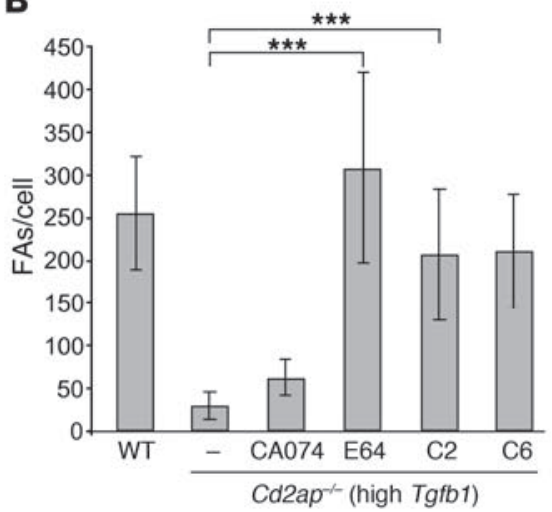

Figure 4

Number of FAs within WT podocytes and high-Tgfb1 Cd2ap-1podocytes with CatL downregulation by diverse treatments. (A) Organization of the actin cytoskeleton and FAs in podocytes in which CatL was downregulated. FAs and F-actin were visualized with anti-paxillin and rhodamine-phalloidin, respectively. (B) Quantitation representing measurements of $>50$ cells shown in $\mathbf{A}$. Data are mean \pm standard deviation. ${ }^{* \star \star} P<0.001$. Scale bars: $20 \mu \mathrm{m}$. downregulation of dendrin resulted in downregulation of Ctsl mRNA and CatL protein (Figure 5, E and F). Furthermore, downregulation of dendrin also partially lowered CatL activity in the cytoplasm of high-Tgfb1 Cd2ap-/- cells (Figure 5G). In agreement with the decrease in CatL activity, downregulation of dendrin also increased the levels of dynamin and synaptopodin in high-Tgfb1 Cd2ap ${ }^{-/-}$cells (Figure 5F).

As shown previously (18), high-Tgfb1 $C d 2 a p^{-/-}$cells were hypersensitive to proapoptotic signals such as high levels of TGF- $\beta 1$, staurosporine, actinomycin D, or angiotensin II (Figure $5 \mathrm{H}$ ). We thus examined whether downregulation of dendrin and/or CatL has functional consequences on $C d 2 a p^{-/-}$podocyte survival. Importantly, downregulation of dendrin in high-Tgfb1 $\mathrm{Cd} 2 \mathrm{ap}^{-/-}$cells resulted in partial protection from TGF- $\beta 1$-induced apoptosis (Figure 5I). This partial rescue may be due to partial downregulation of cytosolic CatL activity (Figure 5, F and G), and thus only partial rescue of the actin cytoskeleton, in high-Tgfb1 $\mathrm{Cd} 2 \mathrm{ap} \mathrm{p}^{-/-}$cells (Figure 5, C-F), or possible additional roles of nuclear dendrin in addition to regulation of CatL expression. In support of the first hypothesis, downregulation of CatL or addition of E64 resulted 


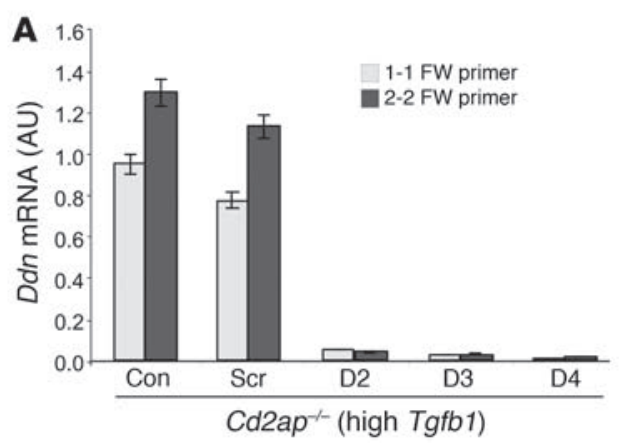

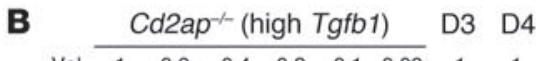
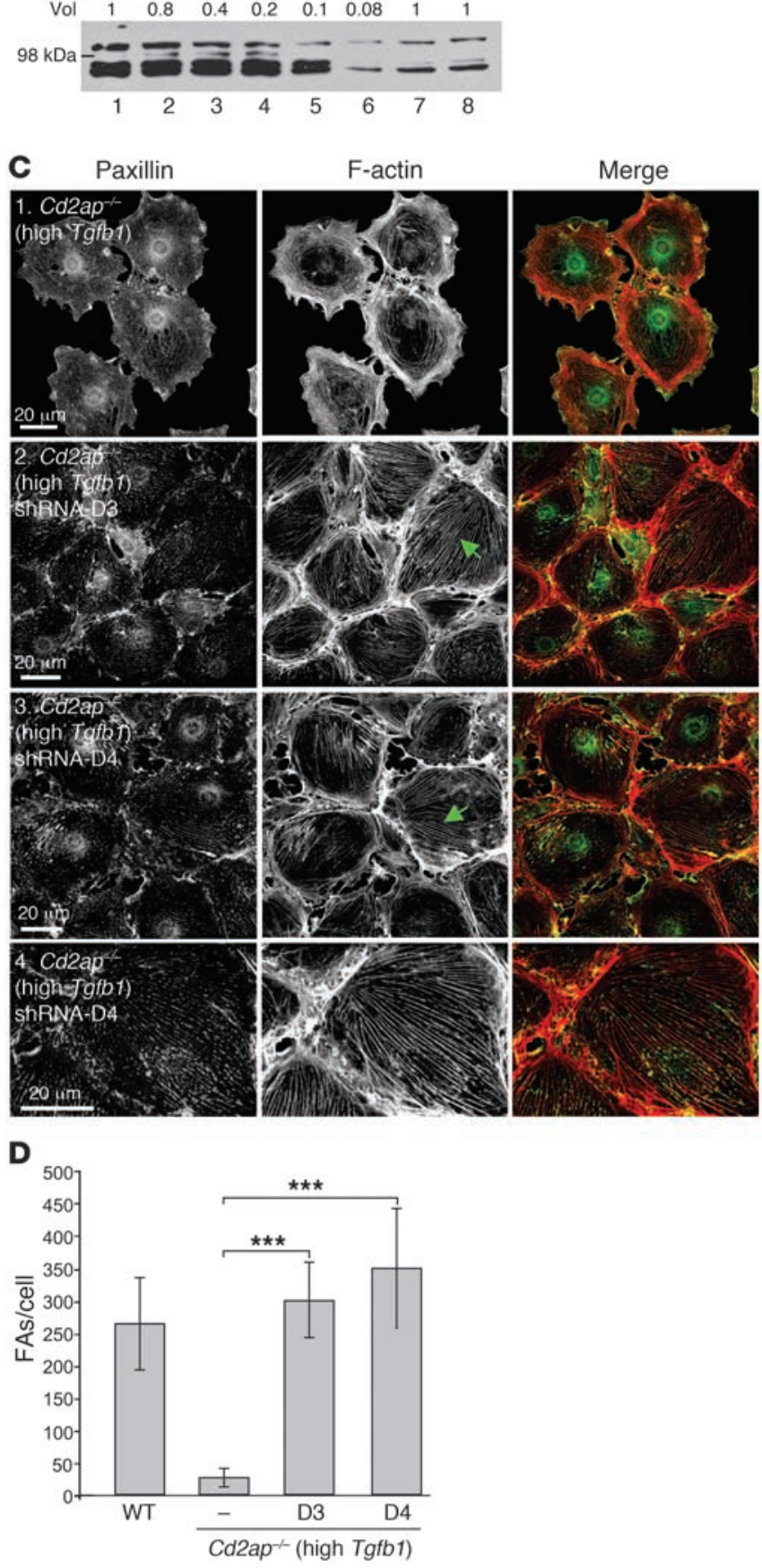
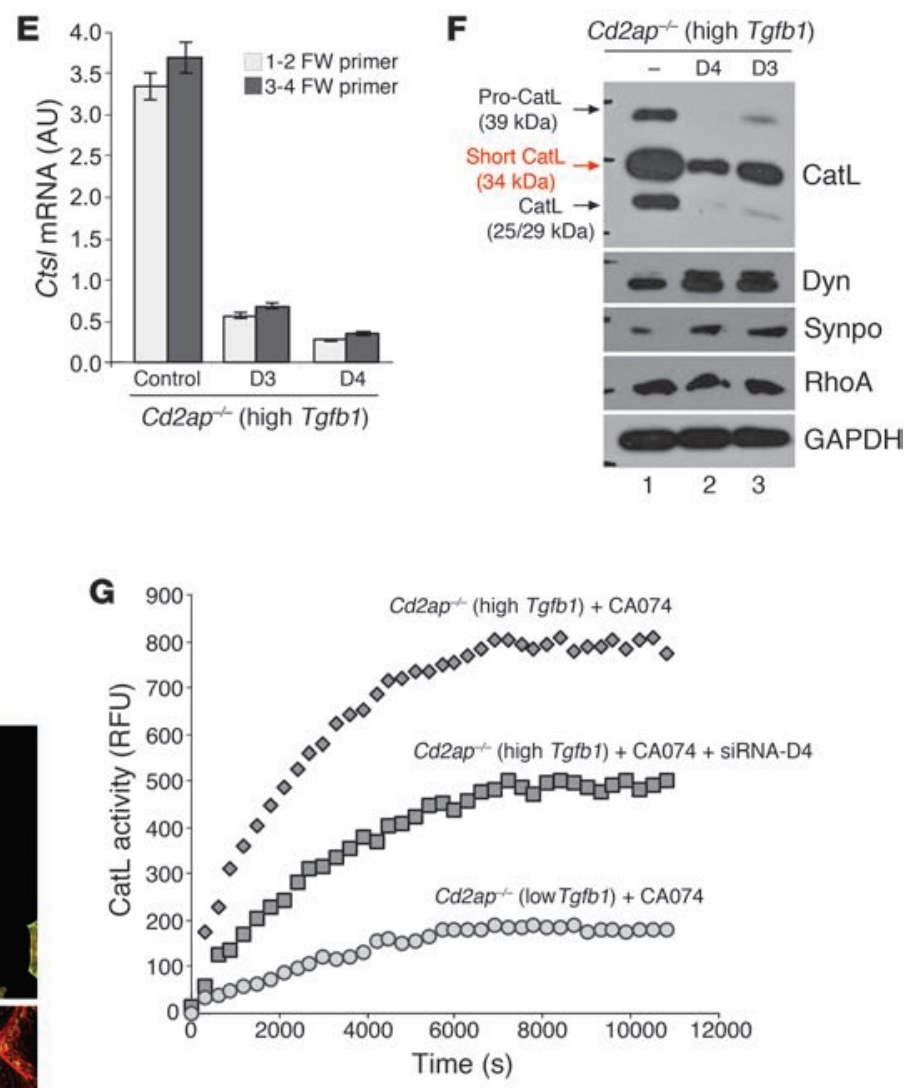

H
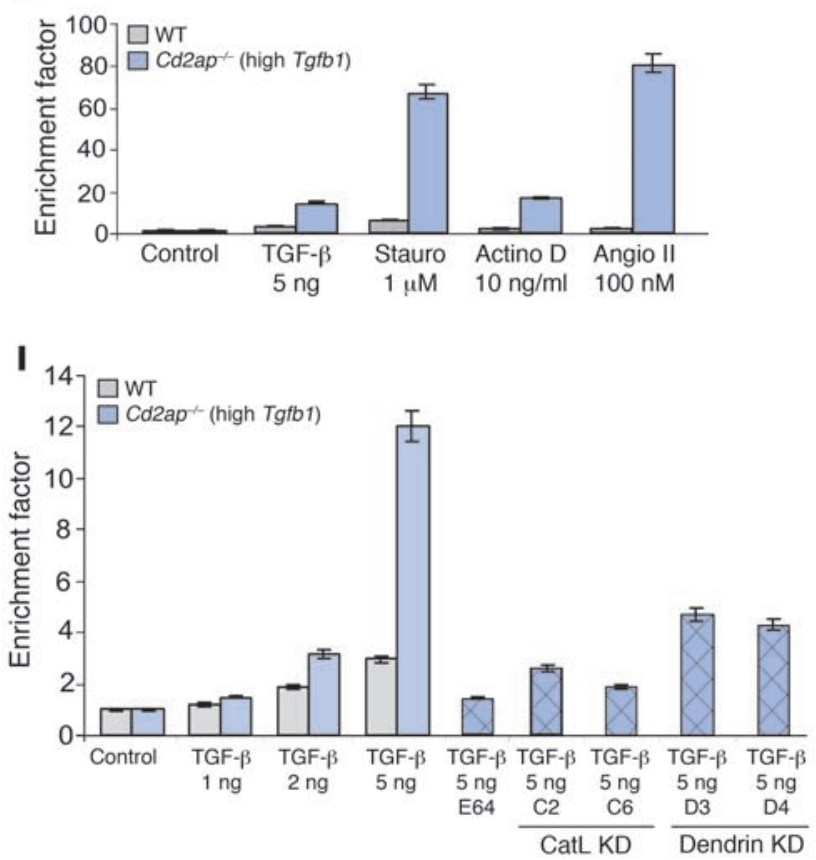


\section{Figure 5}

Prolonged CD2AP loss leads to dendrin translocation into the nucleus. (A) Ddn mRNA levels, determined by RT-PCR, in high-Tgfb1 Cd2ap ${ }^{-/-}$podocytes infected with different shRNA constructs (D2-D4) downregulating endogenous dendrin. Con, uninfected high-Tgfb 1 Cd2ap ${ }^{-/-}$podocytes; Scr, high-Tgfb1 $\mathrm{Cd}_{2} \mathrm{ap}^{-/-}$podocytes infected with lentiviruses expressing a scrambled oligo. (B) Dendrin levels in high-Tgfb1 $\mathrm{Cd}_{2 \mathrm{ap}}{ }^{-/-}$podocytes infected with lentiviruses expressing the 2 most efficient shRNA constructs, D3 and D4. (C) Organization of the actin cytoskeleton and FAs in high-Tgfb1 $\mathrm{Cd}_{2} \mathrm{ap}^{-/-}$podocytes with dendrin downregulation. FAs and F-actin were visualized with anti-paxillin and rhodamine-phalloidin, respectively. (D) Number of FAs within WT and high-Tgfb1 Cd2ap ${ }^{-/}$podocytes with dendrin downregulation. Data (mean \pm standard deviation) represent measurements of $>50$ cells in $\mathbf{C} .{ }^{* *} P<0.001$. (E) Cts/ mRNA levels, determined by RT$\mathrm{PCR}$, in high-Tgfb1 Cd2ap ${ }^{-/-}$podocytes with dendrin downregulation. (F) Protein levels of CatL, dynamin, synaptopodin, RhoA, and GAPDH in high-Tgfb1 $\mathrm{Cd}_{2 \mathrm{ap}} \mathrm{p}^{-1}$ podocytes and cells infected with lentiviruses. (G) Time course of CatL activity in high-Tgfb1 Cd2ap-/- podocytes, high-Tgfb1 $\mathrm{Cd}_{2} \mathrm{ap}^{-/-}$podocytes infected with lentiviruses, and lowTgfb1 $\mathrm{Cd}_{2} \mathrm{ap}^{-/-}$podocytes at neutral $\mathrm{pH}$. (H) Loss of CD2AP rendered podocytes hypersensitive to proapoptotic signals, as shown by specific enrichment of mono- and oligonucleosomes released into the cytoplasm of WT and high-Tgfb1 Cd2ap ${ }^{-/-}$podocytes treated with different apoptosis inducers. Stauro, staurosporine; Actino D, actinomycin D; Angio II, angiotensin II. (I) TGF- $\beta 1$-induced apoptosis was reversed by downregulation of CatL or dendrin in high-Tgfb1 Cd2ap ${ }^{-/}$podocytes. Scale bars: $20 \mu \mathrm{m}$.

in complete protection from TGF- $\beta 1$-induced apoptosis of highTgfb1 Cd2ap $p^{-/}$cells (Figure 5I). Downregulation of dendrin or CatL protected high- $T g f b 1 \mathrm{Cd} 2 \mathrm{ap}^{-/-}$cells only from apoptosis induced by TGF- $\beta 1$, not from other apoptotic signals (Supplemental Figure 3, A-C). Taken together, our results demonstrated that the specific proapoptotic effect of TGF- $\beta 1$ on high-Tgfb1 $C d 2 a p^{-/-}$cells is the result of dendrin translocation into the nucleus, where it regulates expression of cytosolic CatL.

Dendrin is a CatL transcription factor. Our results thus far identified a correlation between the presence of dendrin in the nucleus of podocytes and the expression of cytosolic CatL. We therefore examined whether dendrin can directly regulate the expression of CatL by acting as its transcription factor. First, we examined whether heterologous expression of dendrin in HEK 293 cells can enhance transcriptional activity of the CatL promoter. Using triple transfection experiments in HEK 293 cells, we expressed a plasmid encoding for secreted alkaline phosphatase (SEAP) under the control of the rat CatL promoter, dendrin, and Metridia luciferase under the control of a constitutively active promoter to normalize for cell number and transfection efficiency. Compared with the empty vector, dendrin induced normalized SEAP activity 5 -fold (Figure 6, A and B). The transcriptional activity of dendrin was completely abolished by mutation of its nuclear localization signal (Figure 6, A and B), demonstrating that nuclear translocation of dendrin is essential for mediating its effect on CatL transcription (20). In contrast, CatB promoter activity was lower at baseline and was not inducible by dendrin (Figure 6, A and B), demonstrating specificity of dendrin for CatL. We thus speculated that dendrin might directly bind to the CatL promoter to act as a transcription factor. To identify the dendrin-binding site on the CatL promoter, we studied the effect of dendrin on SEAP transcription from partial deletion constructs of the CatL promoter, containing either bp $-1,224$ to -49 or bp -409 to -49 (Figure $6 \mathrm{C}$ ). Whereas the former construct did not significantly differ from the full-length promoter, the latter lost baseline activity as well as its inducibility by dendrin (Figure 6B), thus mapping the putative dendrin binding site to a region between $-1,224$ and -409 of the CatL promoter. Using EMSA with DNA fragments of decreasing length, we detected direct binding of dendrin to the CatL promoter and fine-mapped the dendrin binding site to a 24-bp region (Figure 6, D and E). Introduction of a 3 -bp mutation in the $5^{\prime}$-terminal part of this region, but not in the middle or the $3^{\prime}$-terminal part, completely abolished dendrin binding (Figure 6C). Together, our analyses demonstrated that nuclear dendrin directly binds to a specific region of the CatL promoter and acts as a transcription factor.

Cytosolic CatL proteolytically processes CD2AP. Destruction of the $\mathrm{SD}$ is a common signature event that occurs literally in all proteinuric glomerular diseases (5). Given our observations downstream of CD2AP deletion, we sought to determine whether loss of CD2AP could be the starting point in many renal diseases at the time the SD is affected. One method of CD2AP destruction could be through proteolysis, possibly by CatL, closing the regulatory loop. We therefore tested whether CatL, in addition to dynamin and synaptopodin, might target CD2AP that contains CatL cleavage sites within its AA sequence (data not shown).

To examine whether CatL can proteolyze CD2AP, we generated N-terminal GFP-tagged and C-terminal FLAG-tagged recombinant CD2AP constructs. Incubation of recombinant CD2AP with purified CatL at $\mathrm{pH} 4.5$ and $\mathrm{pH} 5.5$ led to a complete digestion of the protein (Figure 7A), in accordance with unspecific proteolytic activity of CatL at lysosomal $\mathrm{pH}$. However, at neutral $\mathrm{pH} 7.0$, we detected an approximately $55-\mathrm{kDa} \mathrm{N}$-terminal fragment that was detected with anti-GFP or N-terminal anti-CD2AP antibodies (Figure 7A and Supplemental Figure 4). Although the prediction of endopeptidase substrates algorithm (25) predicted 11 putative CatL cleavage sites within the CD2AP protein (data not shown), data from the cleavage assay suggested that CatL recognizes the QPLGS sequence situated between the second and third domains of src homology domain 3 (Figure 7B). To further test the full spectrum of CD2AP cleavage by CatL, additional proteolytic experiments were performed. A 32-kDa C-terminal CD2AP fragment (termed p32) was also detected by C-terminal anti-CD2AP antiserum (Figure 7C, lanes 2-4, and Supplemental Figure 4), suggestive of cleavage through the site LSAAE (Figure $7 D)$. To better define the relevance of these CD2AP cleavage fragments, we tested generation of CD2AP cleavage fragments in cells. For these experiments, we used WT Ctsl mRNA (which generates both lysosomal and cytosolic CatL protein) or a CatL construct that contains a deletion of the first AUG site and thus encodes selectively for cytosolic CatL (10). Cytosolic CatL alone was sufficient to yield p32, which could not be detected using anti-FLAG antibody (Figure 7E). Generation of p32 was prevented by coincubation of transfected HEK 293 cells with E64 (Figure 7E). To determine which cleavage sites are required for $\mathrm{p} 32$ generation, we expressed a C-terminal FLAG-tagged CD2AP fragment $(71 \mathrm{kDa})$ as well as a C-terminal FLAG-tagged CD2AP mutant with deleted LSAAE site (Figure 7, C and D) in HEK 293 cells and immobilized on FLAG beads before digestion with purified CatL enzyme. Whereas WT CD2AP was proteolyzed to $\mathrm{p} 32$, deletion of $L S A A E$ protected it from CatL cleavage (Figure 7F). Of note, the deletion of QPLGS did not prevent generation of p32 (data not shown), which suggests that $L S A A E$ is the more critical site within CD2AP that is being targeted by cytosolic CatL. 
A
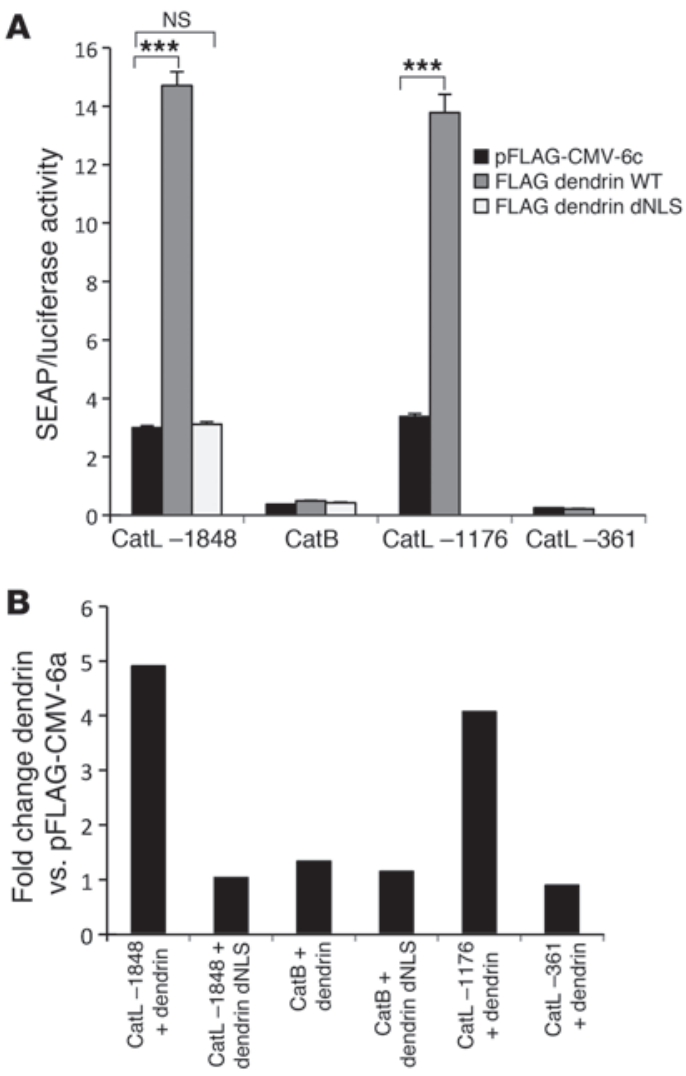

D

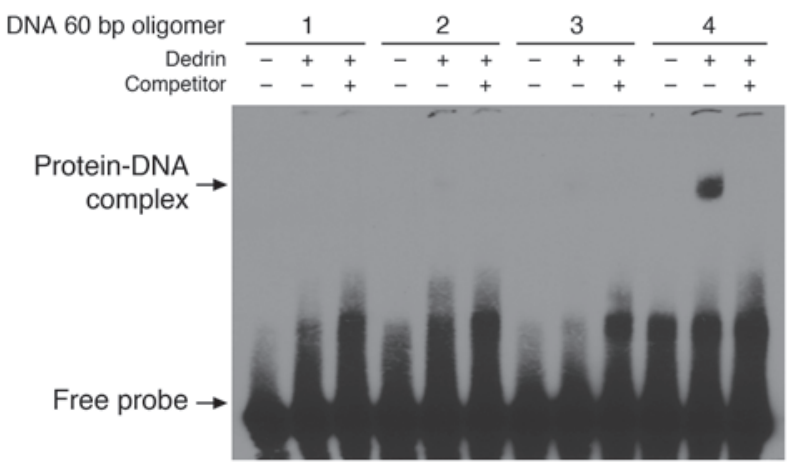

C

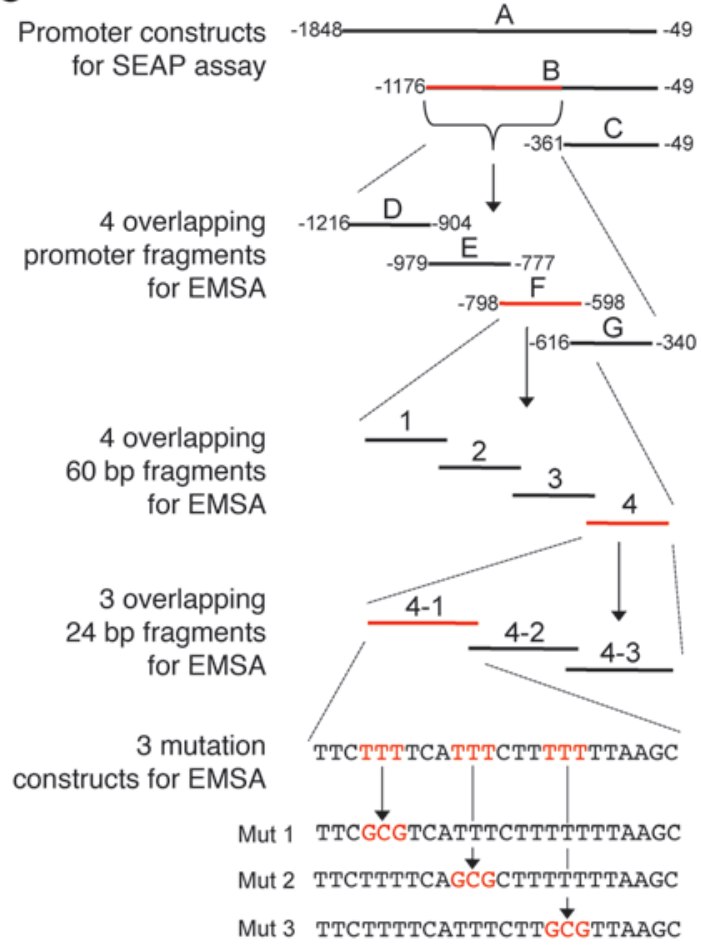

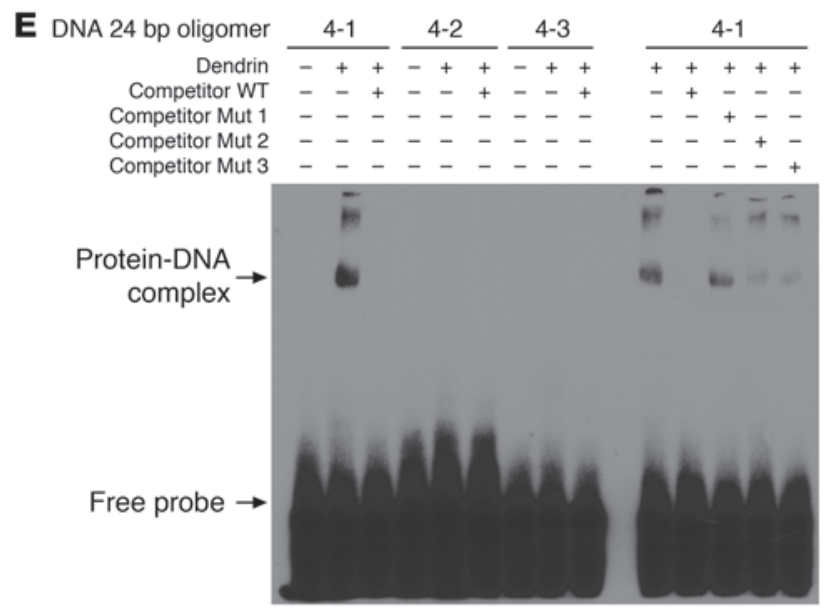

Figure 6

Dendrin is a transcription factor of CatL. (A and B) Nuclear dendrin induced transcription from the CatL promoter, but not from the CatB promoter. HEK 293 cells were cotransfected with pSEAP reporter vector containing the promoter of interest, dendrin, and Metridia luciferase to normalize for transfection efficiency. Mutant dendrin lacking its nuclear localization signal (dNLS) was ineffective. Promoter activity and response to dendrin depended on a region between $\mathrm{bp}-1,215$ and bp -339 , as revealed by partial deletion promoter constructs. ${ }^{* * *} P<0.001$. (C) Strategy used to identify the dendrin binding site within the CatL promoter. The promoter fragment between bp $-1,215$ and -339 was divided into 4 parts. EMSA with these unlabeled DNA fragments, visualized by SYBR green, revealed dendrin binding to fragment $F$ (data not shown), which was then further divided into overlapping fragments to fine-map the dendrin binding site. (D) EMSA demonstrating specific dendrin binding to one of the biotin-labeled 60 -bp oligonucleotides that were completely abolished by a 200 -fold excess of unlabeled oligonucleotide. (E) The 60-bp oligonucleotide that exhibited dendrin binding was divided into 3 overlapping 24-bp oligonucleotides to further narrow the dendrin binding site. Mutation of 3 nucleotides in the 5' region, but not in the central or 3' region, of the 24-bp oligonucleotide 4-1 completely abolished dendrin binding, as demonstrated by the inability of a 200 -fold excess of unlabeled mutated oligonucleotide to compete with the labeled WT oligonucleotide. 
To learn about the functional relevance of partially degraded CD2AP, we analyzed the known interactions that CD2AP undergoes with synaptopodin (16), the SD protein nephrin (26), and dendrin (20). We performed coimmunoprecipitation studies with GFP- and FLAG-tagged protein combinations expressed in HEK 293 cells. Both the $\mathrm{N}$-terminal fragment and p32 were still able to bind synaptopodin and nephrin, but only $\mathrm{N}$-terminal CD2AP bound dendrin (Figure $7 \mathrm{G}$ ). These data suggest that loss of CD2AP as a result of proteolysis might trigger a cellular cascade similar to that in $\mathrm{Cd} 2 \mathrm{ap}^{-/-}$podocytes, which is characterized by the dendrin translocation into the nucleus. In line with the biochemical data, we detected loss of protein levels for CD2AP in podocytes treated with LPS (Supplemental Figure 5A), which is known to increase cytosolic CatL activity (6). Downregulation of CD2AP was also observed in the soluble fraction of isolated glomeruli from mice treated with LPS (Supplemental Figure 5B). There was a decrease in staining for CD2AP in glomeruli of LPS-treated mice (Supplemental Figure 5, C and D). Finally, in agreement with our data in cells (Figure 3, C and D), there was an increase in CatL activity in soluble extracts generated from isolated glomeruli of LPS-treated mice (Supplemental Figure 5E). Together, these data provide further evidence that activity of cytosolic CatL underlies podocyte injury through degradation of CD2AP. The LPS model, in contrast to the $C d 2 a p^{-/-}$model (Figure $1 \mathrm{G}$ ), was reversible, as CatL induction was transient (6) and without significant podocyte TGF- $\beta 1$ induction (data not shown). Thus, time-limited LPS-induced CatL expression and subsequent downregulation of CD2AP did not lead to significant dendrin translocation into the nucleus (Supplemental Figure 5F), which might explain the reversibility of the process. This is in line with dendrin knockout mice developing proteinuria after LPS treatment, as the initial CatL increase was dendrin independent (Supplemental Figure 5, G and H).

Having analyzed the critical role of CD2AP as gatekeeper of the TGF- $\beta 1$ response in podocytes, we analyzed CD2AP expression in the human progressive glomerular disease focal segmental glomerulosclerosis (FSGS) and compared it with the nonprogressive minimal change disease (MCD; Figure 7, $\mathrm{H}$ and I). We detected no reduction in CD2AP staining in glomeruli of patients with MCD (Figure 7I), which is known to be caused by angiopoietin-like 4 protein (27) or by increased c-mip expression (28). In contrast, glomeruli in human progressive FSGS, which are known to have increased CatL expression (6), showed a prominent loss of N-terminal CD2AP (Figure 7I) when using the anti-N-terminal CD2AP antisera, but not when using the anti-C-terminal CD2AP antisera (Supplemental Figure 4). Together, these data suggest that loss of $\mathrm{CD} 2 \mathrm{AP}$ (genetic and proteolytic) is a key upstream event in the development of progressive glomerular diseases such as FSGS.

\section{Discussion}

Here we identified a proteolytic program under control of a functional SD. According to this regulatory system, the intact SD requires full-length $\mathrm{CD} 2 \mathrm{AP}$ that keeps transcription factors, such as dendrin, at the plasma membrane (Figure 8). Injury to the SD by mutations in CD2AP (29), genetic deletion (30), or enzymatic destruction allow for translocation of dendrin from the plasma membrane to the nucleus (Figure 8). Nuclear dendrin binds the CatL promoter and turns on sustained expression of cytosolic CatL. CatL-mediated podocyte injury has 2 components: (a) reorganization of the podocyte actin cytoskeleton owed to the proteolytic downregulation of dynamin, synaptopodin, and RhoA (e.g., loss of FAs and stress fibers), which underlies FP effacement, and (b) decreased podocyte survival caused by decreased TGF- $\beta$ threshold. Thus, the injured podocytes become hypersensitive to TGF- $\beta$ proapoptotic signals, and TGF- $\beta$-driven podocyte death promotes progression of kidney diseases. We showed that cytosolic CatL was capable of regulating sustained expression of itself by degrading CD2AP, which explains why restoration of podocyte structure and function in the clinic is often time-limited with more damage to occur as the injury persists. Because podocyte injury is directly linked to proteinuria, it is generally assumed that proteinuria is the cause for increasing kidney damage. Our data, however, argue that a main reason for progressive renal disease lies in the cellular injury mechanism of podocytes, rather than in proteinuria.

Role for TGF- $\beta 1$ signaling in regulating cytosolic CatL expression in podocytes. TGF- $\beta 1$ is a pleotropic cytokine that has been previously implicated in pathogenesis of renal fibrosis and, ultimately, end-stage kidney diseases (21). The TGF- $\beta$ isoforms (TGF- $\beta 1-$ TGF- $\beta 3$ ) are widely expressed and act on virtually every cell type in mammals by engaging a ubiquitous intracellular signaling cascade of Smad family proteins through ligand-induced activation of heteromeric transmembrane TGF- $\beta$ receptor kinases. In addition, TGF- $\beta$ receptors can activate Smad-independent signaling mechanisms, including MAPKs and PI3K (31). However, molecular mechanisms of activation of Smad-independent pathways by TGF- $\beta$ receptors are still not fully defined. It has been shown that loss of the adaptor protein CD2AP leads to increased expression of TGF- $\beta 1$ in podocytes, where its autocrine effects lead to activation of proapoptotic p38MAPK as well as inhibition of the antiapoptotic PI3K/AKT pathway (22). Here, progression to end-stage kidney disease and, ultimately, death in mice lacking CD2AP were attributed to increased TGF- $\beta 1$-mediated podocyte death. Expanding on original studies, we showed that loss of CD2AP in podocytes, and the subsequent increase in TGF- $\beta 1$ expression, led to translocation of the SD protein dendrin from the plasma membrane into the nucleus. Nuclear dendrin then acted as transcription factor for cytosolic CatL. Thus, our study identified yet another role for TGF- $\beta 1$ signaling in podocytes: regulation of CatL expression. Our results suggest that loss of direct interactions between CD2AP and dendrin at the SD facilitated dendrin translocation in a TGF- $\beta 1$-dependent manner (Figure 1, D and $\mathrm{H})$. In agreement with this rationale, high levels of TGF- $\beta 1$ were not sufficient to induce significant dendrin translocation into the nucleus in WT podocytes (Figure 2B), nor did TGF- $\beta 1$ induce high levels of Ctsl mRNA expression (Figure 2D). Our data also showed that the presence of CD2AP protected podocytes from the effects of high levels of TGF- $\beta 1$ signaling with respect to dendrin translocation and, thus, cytosolic CatL expression. Together, these data suggest that CD2AP plays the role of a gatekeeper with respect to effects of TGF- $\beta 1$ signaling on podocytes. This is particularly interesting given that dose-dependent effects of TGF- $\beta 1$ determine podocyte function under various conditions, and losing a controlled cellular intake ultimately results in podocyte death (22). In line with recently published observations in podocytes that link Smad-independent prosurvival signaling of TGF- $\beta 1$ with CD2AP (32), our data suggest that loss of CD2AP switches the cellular balance from prosurvival to proapoptotic via induction of cytosolic CatL. The gatekeeping effect on podocyte TGF- $\beta 1$ by CD2AP was compromised in the face of high intrarenal TGF- $\beta 1$ production (Figure $1 \mathrm{~A}$ ), as this was sufficient to translocate dendrin to the nucleus and increase expression of 
$\begin{array}{lllllllll}\text { A } & \mathrm{pH} & 7.0 & 4.5 & 5.5 & 7.0 & 4.5 & 5.5 & 7.0\end{array}$ GFP-CD2AP ++++++++
CatL -+++++

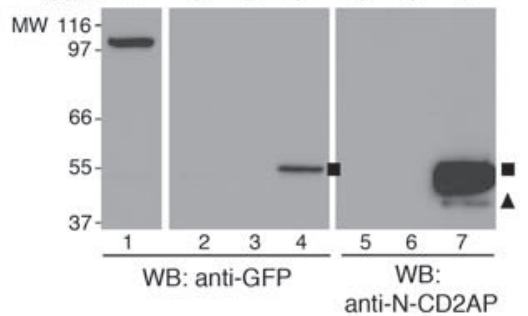

\section{C}

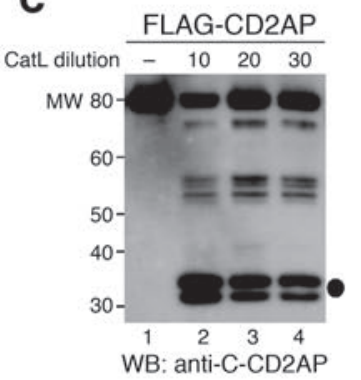

D

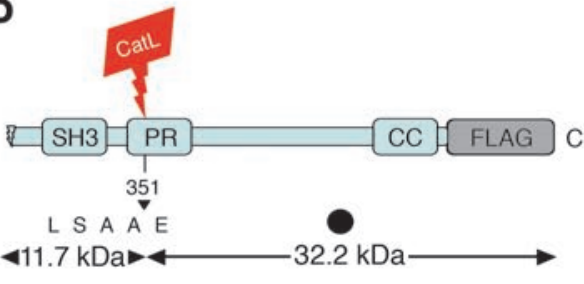

B

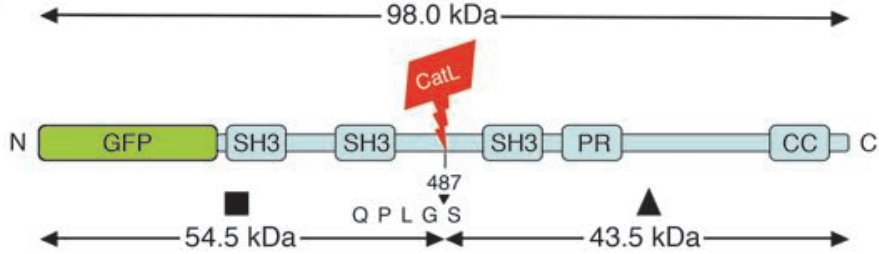

FLAG-

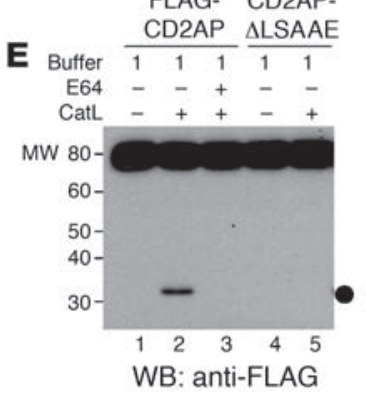

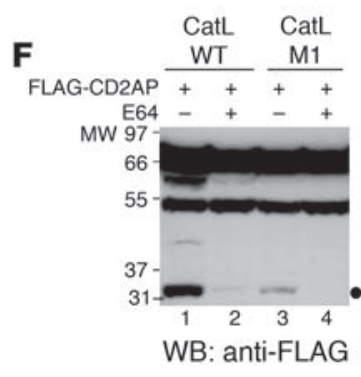

G

IP: FLAG-nephrin IP: FLAG-synpo IP: FLAG-dendrin
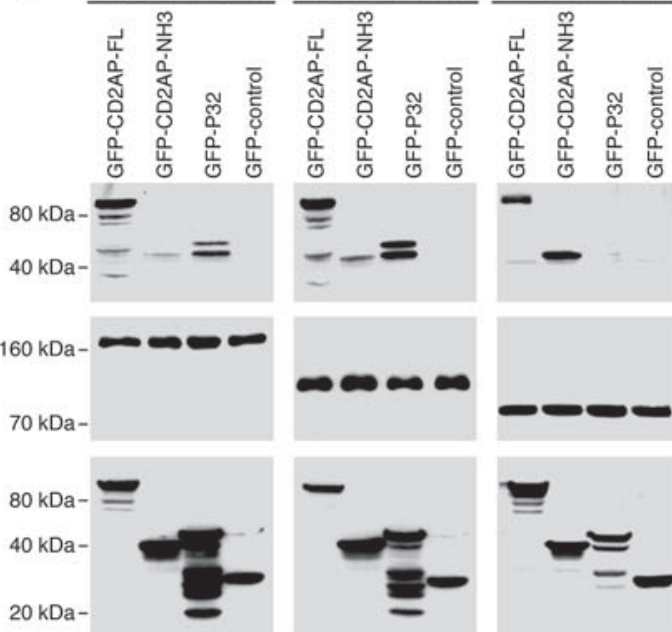

Eluate

WB: anti-GFP Eluate

WB: anti-FLAG

Input

WB: anti-GFP
H

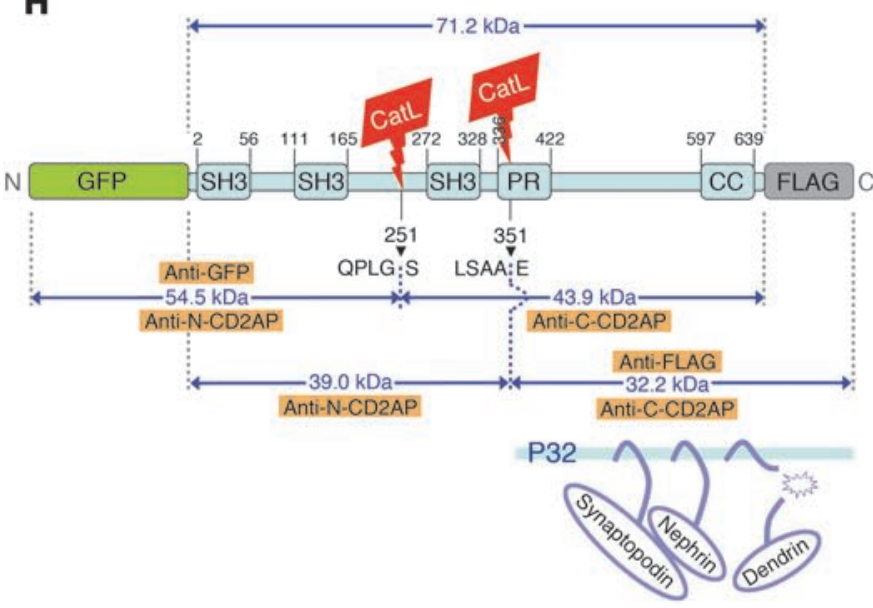

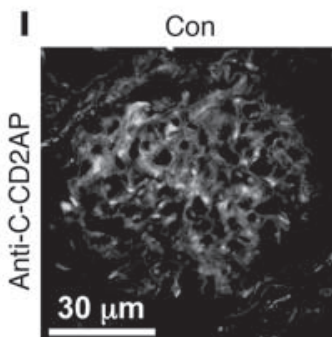
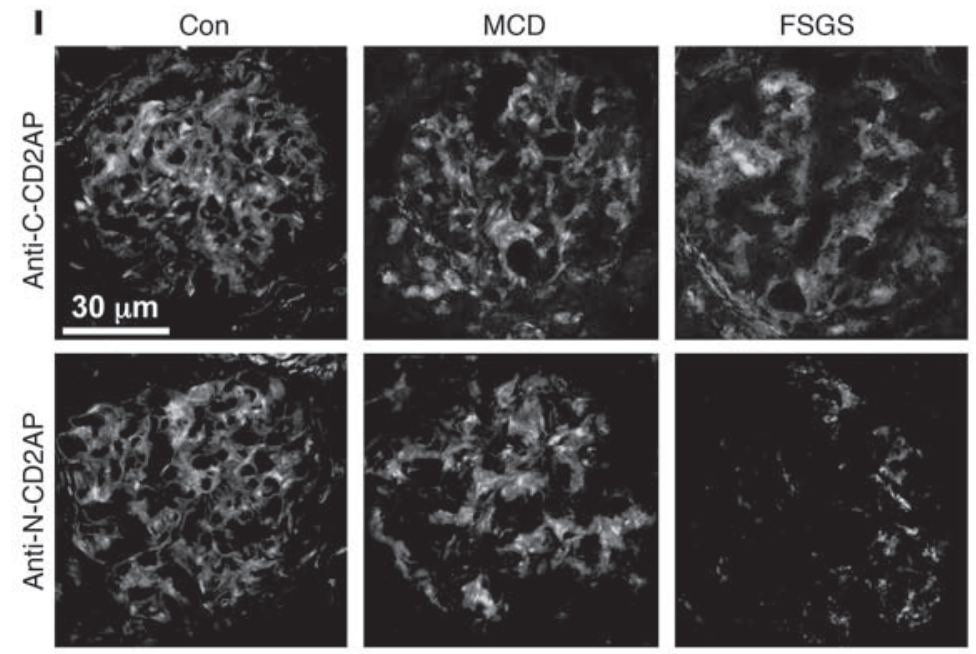


\section{Figure 7}

Cytosolic CatL proteolytically processes CD2AP in podocytes. (A) Immunoblot of cleaved CD2AP fragments tagged with N-terminal GFP. At pH 7.0, CD2AP cleaved into a stable 55-kDa fragment (squares), as detected with anti-GFP antibody. We detect the same fragment with the $\mathrm{N}-\mathrm{CD} 2 \mathrm{AP}$ antiserum. This antiserum also detected a weak band corresponding to a 44-kDa fragment (triangle). (B) Match of cleavage fragments with predicted CatL cleavage site QPLGS. (C) Deletion of the CatL cleavage site $L S A A E$ protected CD2AP from limited proteolysis into p32 (circle). (D) Match of p32 with predicted CatL cleavage site $\angle S A A E$. (E) CatL cleaved CD2AP-FLAG, yielding p32 (circle), detected by anti-C-CD2AP. (F) WT Ctsl cleaved CD2AP in HEK 293 cells. Cytosolic CatL (CatL M1) was sufficient to cleave CD2AP, yielding p32 (circle). These cleavage reactions were prevented by incubation of the cells with E64. (G) Coimmunoprecipitation of nephrin, synaptopodin, and dendrin from HEK 293 cells transfected with full-length CD2AP, $\mathrm{N}$-terminal CD2AP, and p32. (H) Structural domains of CD2AP, together with major CatL cleavage sites, predicted sizes of resulting fragments from CatL digestion, and recognition sites of the antibodies used. SH3, src homology domain 3; PR, proline-rich motif; CC, coiledcoil domain. (I) Immunofluorescent staining of kidney biopsies from patients with MCD and FSGS. N-terminal CD2AP was reduced only in progressive disease (i.e., FSGS). Scale bar: $30 \mu \mathrm{m}$.

CatL. Our data therefore identified a direct link between TGF- $\beta 1$ signaling in podocytes and cytosolic CatL levels.

Given the diversity of pathways that can injure podocytes (33), it seems reasonable to suggest that multiple stimuli can affect podocyte cytosolic CatL levels, in addition to the identified TGF- $\beta 1$ dependent dendrin translocation mechanism. For example, LPSinduced expression of cytosolic CatL appeared to be TGF- $\beta 1$ independent. In addition, CD2AP-dependent signaling at the SD is clearly not the only mechanism that operates in podocytes. Other main proteins involved in signaling at the SD are nephrin and Neph proteins (34). Interactions between nephrin and the adaptor protein Nck have been shown to play a major role in regulation of the actin cytoskeleton during development as well as in the maintenance of healthy, functional podocytes $(2,35,36)$. Our findings identified a parallel signaling pathway from the SD to the actin cytoskeleton, which was mediated by interactions between CD2AP and dendrin.

Role of cytosolic CatL for podocyte apoptosis. We and others have shown that cytosolic CatL degrades dynamin and synaptopodin $(6,7)$. Loss of synaptopodin has been associated with downregulation of RhoA (7). Downregulation of dynamin and RhoA signaling results in loss of stress fibers and FAs, the hallmark of effaced podocytes. We have recently shown that dynamin can regulate actin cytoskeleton in podocytes independently and in parallel to RhoA signaling (37). Thus, it seems as if cytosolic CatL specifically targets both pathways involved in regulating turnover of FAs and stress fibers.

The role of cytosolic CatL in podocyte injury was originally discovered by our laboratories using the LPS injury model (6). Importantly, LPS-induced proteinuria is reversible, and does not lead to progressive glomerular injury (24). Based on the LPS model, FP effacement and proteinuria in itself do not necessarily lead to progressive kidney injury. Together with our present findings, these data suggest that downregulation of dynamin, synaptopodin, RhoA, and CD2AP drives the major reorganization of the actin cytoskeleton, FP effacement, and proteinuria, but not podocyte death. Indeed, high-Tgfb1 $C d 2 a p^{-/-}$podocytes did not exhibit significantly elevated basal level of apoptosis compared with WT podocytes (Figure 5I). However, these cells did exhibit hypersen- sitivity to TGF- $\beta 1$-induced apoptosis. Together, these data suggest that cytosolic CatL-induced FP effacement and proteinuria occur before podocyte death and might represent a repair stage, in which cell structure is given up in order to survive. However, the repair process is timed, because as shown here, effacement was associated with a heightened level of susceptibility to TGF- $\beta 1-$ mediated apoptosis. Thus, the sustained presence of cytosolic CatL, coupled with increased levels of TGF- $\beta 1$ in podocytes, drives podocyte apoptosis and progression to end-stage kidney diseases, as we observed in TGF- $\beta 1 \mathrm{Tg}$ and $\mathrm{Cd} 2 \mathrm{ap}^{-/-}$mice (Figure $1, \mathrm{~B}-\mathrm{H}$ ). In contrast, if injury signal does not lead to increase in TGF- $\beta 1$ signaling in podocytes, as in the case of reversible LPS-induced proteinuria, podocytes have the capability to switch off CatL expression and restore cellular structure and function.

This model was corroborated with the observation of high levels of Ctsl mRNA and CatL protein, as well as degraded CD2AP (Figure 7, $\mathrm{H}$ and $\mathrm{I}$ ), in patients with diverse progressive kidney diseases such as FSGS, diabetic nephropathy, and membranous nephropathy, but not in those with MCD (6). Our study suggests that progression to CKD is most likely driven by the sustained presence of cytosolic CatL.

Role of dendrin in progressive kidney diseases. Although the presence of dendrin in the nucleus has been associated with a proapoptotic phenotype, its exact role in this process has not yet been identified (20). Our results argue for a direct correlation between the presence of nuclear dendrin and the expression of cytosolic CatL. It has previously been shown that expression of lysosomal and extracellular CatL is regulated by transcription factors such as $\mathrm{ZHX}$ proteins (38). Our results identified dendrin as a transcription factor specifically driving expression of cytosolic CatL. The dendrin-dependent increase in Ctsl mRNA in podocytes translated to a dramatic increase in cytosolic CatL expression, without increased levels of lysosomal CatL (Figure 2, E and F). The molecular mechanism that couples increased transcription by dendrin with initiated translation from the downstream AUG sites has not be identified, but is clearly operational in podocytes. What mechanism switches off CatL expression, as in a case of LPS-induced CatL expression, remains an open question that needs to be pursued in the future.

Regardless of the exact mechanism by which dendrin drives expression of cytosolic CatL, the surprising discovery that dendrin was a transcription factor for CatL uncovered the physiological connection between the SD (i.e., CD2AP) and the regulation of podocyte cytoskeleton as well as cellular survival. This functional proteolytic system ties the structure of podocytes with their survival properties in which the input level of TGF- $\beta 1$ acts as a modifier. Together, our data establish the mechanistic base for progressive podocyte disease and provide a rationale as to why proteinuric kidney diseases are generally more prone to progression and podocyte depletion (39). Since TGF- $\beta 1$ not only has deleterious effects on podocytes, but is also part of physiological responses, one could assume that at normal levels, it allows some dendrin to activate the Cat $\mathrm{L}$ promoter to produce small amount of cytosolic CatL that is present to help regulate the podocyte physiological dynamic of the FP cytoskeleton (Supplemental Figure 2 and refs. 6 and 7). However, persistent high TGF- $\beta 1$ input into podocytes likely drives aggravating glomerular injury through dendrin translocation. This notion is underscored in the recent study by Asanuma and coworkers, in which they observed dendrin-positive podocyte nuclei in progressive experimental and human glomerular diseases (40).

Our study showed that downregulation of dendrin or CatL was sufficient to overcome TGF- $\beta 1$-mediated susceptibility to apopto- 
Healthy podocyte

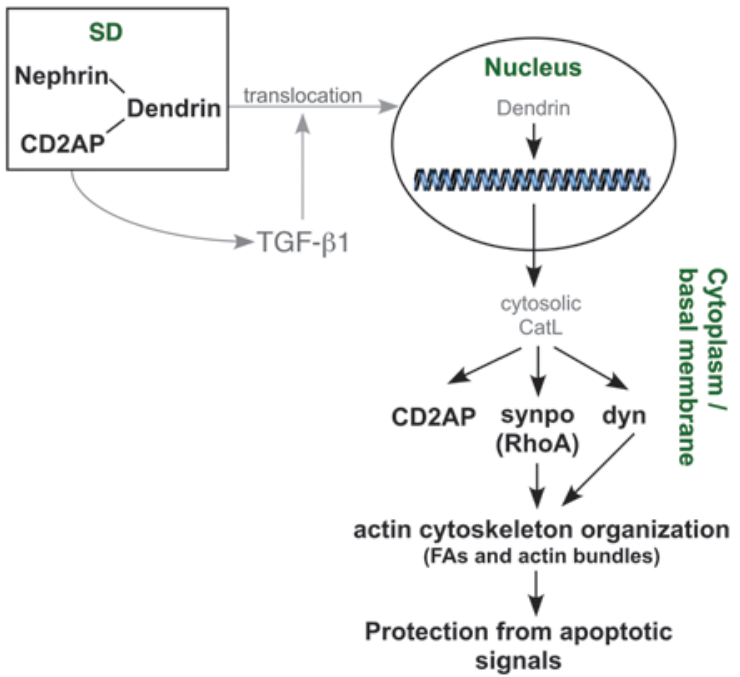

Injured podocyte

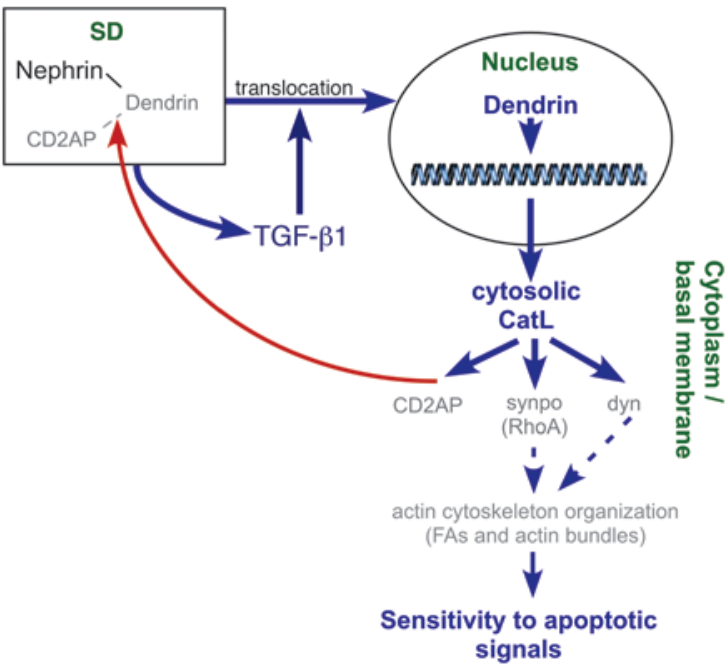

Figure 8

Signaling between nucleus and cytoplasm of healthy and injured podocyte and SDs are mediated by CatL.

sis. In agreement with our findings, stabilizing the phenotype in $C d 2 a p^{-1-}$ mice can be achieved by crossing them with mice deficient in dendrin, resulting in delayed proteinuria onset and improved survival (41), which suggests that the events of FP effacement and podocyte apoptosis can be separated by specific interventions. Our findings allow for the conceptualization of strategies for renal protection that work in concert with antiproteinuric modalities by aiming at rescuing podocyte survival. Combining both approaches will likely result in improved human health.

\section{Methods}

Cell culture and transient transfection. Mouse WT and Cd2ap-/- (18) podocytes were cultured as described previously (42). HEK 293 cells were maintained and transfected using Lipofectamine 2000 reagent (Invitrogen) as previously reported (6). Adenoviral infections of cultured podocytes were preformed as described previously (6).

Lentiviral infection. Lentiviral shRNA plasmids for CatL were obtained from Open Biosystems and were used to generate lentiviral transduction particles in HEK $293 \mathrm{~T}$ cells. We used a target set of 3 clones with pLKO.1<-puro as the parental vector (see Supplemental Table 1 for sequences). Lentiviral shRNA plasmids for dendrin were obtained from Sigma Aldrich and were used to generate lentiviral transduction particles in HEK 293 T cells. We used a target set of 2 clones with pLKO.1<-puro as a parental vector (Supplemental Table 1). Lentiviral knockdown of mouse CatL and dendrin were performed in differentiated mouse and high-Tgfb1 $C d 2 a p^{-/-}$podocytes according to the protocol from the RNAi Consortium. Cells were harvested to assay for knockdown efficiency using quantitative PCR and Western blot. In addition, changes in phenotype were monitored using immunofluorescence.

Antibodies. The following primary antibodies were used: mouse anti-actin (Sigma-Aldrich), rhodamine- and FITC-phalloidin (Invitrogen), mouse anti-dynamin, mouse anti-paxillin (Millipore), rabbit anti-WT1, rabbit anti-RhoA, goat anti-synaptopodin (Santa Cruz Biotechnology), mouse anti-GAPDH, rabbit anti-mannosidase II (Abcam), rabbit anti-CD2AP (43), mouse anti-synaptopodin (44), rabbit anti-dendrin (20), rabbit anti-CatL (45), rabbit anti- $\alpha$-actinin-4 (46), rabbit anti-nephrin (47), and rat anti-
Lamp-2 (Developmental Studies Hybridoma Bank). CatL epitope was generated in house, and cytosolic CatL antibody production was outsourced to Picono Rabbit Farm and Laboratory.

Animals and treatments. TGF- $\beta 1 \mathrm{Tg}$ mice (48) and $C d 2 a p^{-/-}$mice on a mixed C57BL6/129J background (12) were described previously. Dendrin knockout mice were generated by replacing the entire coding region of 2 exons with EGFP lox-Ub1-EM7-Neolox cassette (Regeneron Pharmaceuticals). The elimination of dendrin in knockout mice was confirmed by immunohistochemistry (49). The mouse model of LPS-induced proteinuria was as previously described (50). The rat puromycin aminonucleosideinduced nephrosis model was as previously described (51). Urine microalbumin was assessed by densitometric analysis of Bis-Tris gels (Invitrogen) loaded by standard BSA (Bio-Rad Laboratories) and urine samples. The urine creatinine measurement was carried out using a colorimetric endpoint assay with a commercial kit (Cayman Chemical). Animal protocols and procedures were reviewed for ethical and humane standards and approved by the institutional Animal Care Committees of Massachusetts General Hospital and University of Miami.

Isolation and processing of glomeruli. Glomeruli were isolated from kidneys of 8- to 12-week-old LPS- and PBS-treated (control) mice using a sequential sieve technique with mesh sizes of 180,100 , and $71 \mu \mathrm{m}$. The fraction collected from the $71-\mu \mathrm{m}$ sieve was maintained for soup/pellet fractionation as previously described (6). Isolated glomeruli were homogenized in CHAPS buffer containing $20 \mathrm{mM}$ Tris ( $\mathrm{pH} 7.5$ ), $500 \mathrm{mM} \mathrm{NaCl}, 0.5 \%$ (w/v) CHAPS, and protease inhibitors (Roche) using Dounce homogenizer. Subsequently, the extract was centrifuged for 10 minutes at $15,000 \mathrm{~g}$.

Isolation of nuclear fraction. Podocytes were detached, precipitated, and washed with PBS. Cell pellets were immediately resuspended in $400 \mu \mathrm{l}$ chilled buffer A (10 mM HEPES, pH 7.9; 10 mM KCl; 0.1 mM EDTA; $0.1 \mathrm{mM}$ EGTA; $1 \mathrm{mM}$ dithiothreitol [DTT]; and $0.5 \mathrm{mM}$ PMSF). This resuspension mixture was incubated on ice for 15 minutes without vortexing, and then $25 \mu \mathrm{l}$ of $10 \% \mathrm{NP}-40$ was added followed by vigorous vortexing for 10 seconds at $4^{\circ} \mathrm{C}$. The sample was centrifuged at $1,500 \mathrm{~g}$ for 1 minute at $4^{\circ} \mathrm{C}$. The resulting supernatant was collected and stored as the cytosolic fraction. The remaining nuclear pellet was resuspended in $50 \mu \mathrm{l}$ ice-cold buffer B (20 mM HEPES, pH 7.9; $400 \mathrm{mM} \mathrm{NaCl} ; 1 \mathrm{mM}$ 
EDTA; 1 mM EGTA; $1 \mathrm{mM}$ DTT; and $0.5 \mathrm{nM}$ PMSF), rocked vigorously at $4^{\circ} \mathrm{C}$ for 2 hours, and then centrifuged at maximum speed for $10 \mathrm{~min}$ utes at $4{ }^{\circ} \mathrm{C}$. The nuclear and cytosolic fractions were separated by $12 \%$ SDS-PAGE, transferred on a PVDF membrane (Bio-Rad Laboratories), and analyzed by Western blotting.

Immunohistochemistry, immunofluorescence, and immunoblotting. Mouse and rat kidney tissue was harvested and either immersion-fixed in formaldehyde (Electron Microscopy Sciences) for paraffin embedding or embedded in OCT compound (Sakura Finetek) for frozen sections. Sections of paraffin-processed kidney were stained with H\&E. The protocol to study kidney biopsy samples from FSGS and MCD patients was reviewed and approved with a consent waiver by the University of Miami Institutional Review Board. Kidney biopsies were stained with $\mathrm{N}$ - and C-terminal CD2AP antibodies according to standard protocols (52). Immunofluorescence of cultured podocytes and Western blotting were performed as described previously (6). Images were captured using a LSM 5 PASCAL laser scanning microscope (Zeiss) and a $\times 40$ objective. Quantification of stress fibers, FAs, CatL, and hematoxylin immunostain intensities were performed using ImageJ software.

Coimmunoprecipitation studies. The recombinant mouse FLAG-dendrin and GFP-tagged CD2AP variants (full-length $\mathrm{CD} 2 \mathrm{AP}, \mathrm{CD} 2 \mathrm{AP}-\mathrm{NH}_{2}$, and $\mathrm{CD} 2 \mathrm{AP}-\mathrm{COOH}$ ) were expressed in HEK 293 cells. FLAG fusion proteins were immunoprecipitated from cell lysates using anti-FLAG-M2 beads (Sigma-Aldrich), and eluates were analyzed by immunoblotting using antibodies to FLAG (Sigma-Aldrich) or GFP (Invitrogen).

Endopeptidase cleavage site score. To assess the susceptibility of CD2AP for cleavage by CatL in silico, we used the prediction of endopeptidase substrates (PEPS) bioinformatics tool (25). A score above the threshold of 0.01 estimates protein sequences to be within 100 peptide motifs (of 10,000).

Purification of CD2AP and dendrin. FLAG-CD2AP and FLAG-dendrin were expressed in HEK 293 cells, immobilized on anti-FLAG M2 agarose (SigmaAldrich), and eluted with FLAG peptide (Sigma-Aldrich).

Proteolytic processing of CD2AP by CatL. CD2AP was diluted in buffer containing $200 \mathrm{mM} \mathrm{NaCl}, 10 \mathrm{mM}$ HEPES ( $\mathrm{pH} 7.0$ ), 2 mM EGTA, $1 \mathrm{mM} \mathrm{MgCl}_{2}$, and $1 \mathrm{mM}$ DTT. When indicated, $20 \mu \mathrm{M}$ CatL inhibitor I (Calbiochem) was added. The reaction was initiated by addition of $0.5 \mu \mathrm{l}$ purified CatL enzyme (Sigma-Aldrich), and samples were placed at $37^{\circ} \mathrm{C}$ in a water bath for 10-30 minutes. Total assay volume was $20 \mu \mathrm{l}$. The reaction was terminated with addition of $\times 4$ sample buffer (Invitrogen).

Deletion of CD2AP cleavage site LSAAE. Deletion of the CatL cleavage site $L S A A E$ from the CD2AP amino acid sequence was done using the QuickChange II Site Directed Mutagenesis kit (Stratagene) according to the manufacturer's instructions.

Quantitative PCR. Cells were treated with TRIzol reagent (Invitrogen) to allow complete cell lysis, followed by RNA extraction per the manufacturer's protocol. RNA was quantitated and cDNA synthesis was performed using the Protoscript First strand cDNA synthesis kit (New England Biolabs). Quantitative PCR was performed using Brilliant SYBR Green Master Mix (Stratagene) and specific primers for CatL, dendrin, dynamin, RhoA, and synaptopodin (Supplemental Table 2) in MX3000P QPCR System (Agilent Technologies). Normalization across samples was performed using the average of the constitutive gene Gapdh. Fold expression changes were determined using the comparative CT method for relative quantification with the calculation $2^{-\Delta \Delta C T}$, and data were graphed using either Excel (Microsoft) or Prism (Graph-Pad) software.

Kidney total RNA isolation and quantitative RT-PCR. Harvested mouse kidneys were homogenized in TRIzol reagent (Invitrogen) for 40 seconds using PowerGen125 (Fisher Scientific) at maximum speed. Total RNA was isolated according to the manufacturer's protocol. Quality and quantity of total RNA was checked by Bio-analyzer (Agilent Technologies). $1 \mu \mathrm{g}$ kidney total RNA was reversely transcribed into single-strand cDNA. Quantitative
RT-PCR was performed as described previously (53). Expression of GAPDH and $\beta$-actin was used to normalize the sample amount.

$C a t L$ activity assays. Prior to enzyme assays, cytosolic fraction containing CatL was isolated by subcellular fraction as described previously (54). Activity assays were performed using the CatL and CatB fluorescent substrate Z-Phe-Arg-7-amido-4methylcoumarin hydrochloride (Sigma-Aldrich) at different pHs. $5 \mu \mathrm{l}$ supernatant (fixed protein concentration) from each sample was added in triplicate to a 96-well, all-black flat-bottomed plate, followed by addition of $175 \mu \mathrm{l}$ freshly made assay buffer $(340 \mathrm{mM}$ sodium acetate, $60 \mathrm{mM}$ acetic acid, $4 \mathrm{mM}$ disodium EDTA, $8 \mathrm{mM}$ dithiothreitol). The CatB-specific inhibitor CA074 (Enzo Life Sciences) was added to the wells to cancel out the contribution of CatB. The cysteine protease inhibitor E64 (Biomol) was added to the wells designated for negative control. The mixture was incubated at room temperature for 1 minute to activate the enzyme, immediately followed by the addition of $25 \mu \mathrm{l}$ of $20 \mu \mathrm{M}$ fluorescent substrate. Fluorescence of free aminomethyl coumarin was determined as a kinetic interval assay, with readings taken every 5 minutes for 3 hours at $30^{\circ} \mathrm{C}$ by excitation at $370 \mathrm{~nm}$ and emission at $460 \mathrm{~nm}$ using a SpectraMax M2E (Molecular Devices). Data were collected every 5 minutes.

SEAP reporter assay. HEK 293 cells were triple-transfected using Lipofectamine 2000 reagent (Invitrogen) with the following 3 plasmids: (a) pSEAP2-Basic, containing either the full-length rat CatL promoter (construct A; Figure 6C), 1 of 2 partial deletion constructs (constructs B and C; Figure 6C), or the full-length CatB promoter (all gifts of S.S. Chugh, University of Alabama, Birmingham, Alabama, USA; ref. 38); (b) WT rat dendrin, rat dendrin with a mutated nuclear localization signal (20), or the empty vector; and (c) pMetLuc-Control for normalization (Clontech). All transfections were performed in triplicate. Medium was analyzed for SEAP and luciferase activity according to the manufacturer's instructions in a GloMax-96 Microplate Luminometer (Promega).

EMSA. 4 overlapping fragments (fragments D-G; Figure 6C) of the rat CatL promoter portion between $-1,215$ and -339 (provided by S.S. Chugh; ref. 38) were incubated with purified FLAG-dendrin for EMSA using the Electrophoretic Mobility Shift Assay kit (Molecular Probes) and visualized with SYBR green. Promoter fragment E, which bound to dendrin, was further divided into 4 overlapping 60-bp oligonucleotides (see Supplemental Table 3 for sequences); oligonucleotide 4 , which exhibited dendrin binding, was divided again into 3 overlapping 24-bp oligonucleotides (Supplemental Table 3), which were biotinylated at the $5^{\prime}$ end and used for EMSA using the LightShift Chemiluminescent EMSA kit (Thermo Scientific). Corresponding unlabeled WT oligonucleotides were used for competition in 200-fold excess. Finally, oligonucleotide 4-1, which exhibited dendrin binding, was used to design 3 different mutant oligonucleotides (see Figure 6C for sequences) that were used for competition assays in 200 -fold excess.

Statistics. Statistical analysis was performed by 2 -tailed Student's $t$ test. A $P$ value less than 0.05 was considered significant. Unless otherwise indicated, data are reported as mean \pm SEM.

\section{Acknowledgments}

We thank Kirk Campbell (Mount Sinai School of Medicine, New York, New York, USA) for helpful discussions; Livia S. Gandhi (Massachusetts General Hospital, Boston, Massachusetts, USA) for her help with cell culture; and Dayami Hernandez and Cristina Muresan (University of Miami, Miami, Florida, USA) for assistance with H\&E staining and confocal microscopy, respectively. This work was supported by NIH grants DK073495 and DK089394 (to J. Reiser). C. Wei is the Halpin Scholar of the American Society of Nephrology. C.C. Möller was supported by a scholarship of the German Academic Exchange Service. A. Kistler is supported by grant PBZHP3-128278 from the Swiss National Science Foundation and 
an Amgen-FROMO fellowship. M.M. Altintas was supported by NIH training grant T32DK007540. S. Yaddanapudi was supported by NIH training grant T32DK007540. S. Sever was supported by NIH grant DK087985 as well as by the NephCure foundation. The authors also acknowledge the support of the Katz Family Fund.

Received for publication April 16, 2011, and accepted in revised form July 20, 2011.

1. Kriz W, et al. Tracer studies in the rat demonstrate misdirected filtration and peritubular filtrate spreading in nephrons with segmental glomerulosclerosis. J Am Soc Nephrol. 2001;12(3):496-506.

2. Tryggvason K, Pikkarainen T, Patrakka J. Nck links nephrin to actin in kidney podocytes. Cell. 2006; 125(2):221-224.

3. Kriz W, Lemley KV. The role of the podocyte in glomerulosclerosis. Curr Opin Nephrol Hypertens. 1999;8(4):489-497.

4. Wharram BL, et al. Podocyte depletion causes glomerulosclerosis: Diphtheria toxin-induced podocyte depletion in rats expressing human diphtheria toxin receptor transgene. J Am Soc Nephrol. 2005; 16(10):2941-2952.

5. Tryggvason K, Patraaka J, Wartiovaara J. Hereditary proteinuria syndromes and mechanisms of proteinuria. NEngl J Med. 2006;354(13):1387-1401.

6. Sever S, et al. Processing of the GTPase dynamin by extralysosomal cathepsin $\mathrm{L}$ defines a mechanism for proteinuric kidney disease. J Clin Invest. 2007;117(8):2095-2104.

7. Faul C, et al. The actin cytoskeleton of kidney podocytes is a direct target of the antiproteinuric effect of cyclosporine A. Nat Med. 2008;14(9):931-938.

8. He L, et al. Glomerulus-specific mRNA transcripts and proteins identified through kidney expressed sequence tag database analysis. Kidney Int. 2007;71(9):889-900.

9. Reiser J, Adair B, Reinheckel T. Specialized roles for cysteine cathepsins in health and disease. J Clin Invest. 2010;120(10):3421-3431.

10. Goulet B, et al. A cathepsin L isoform that is devoid of a signal peptide localizes to the nucleus in $\mathrm{S}$ phase and processes the CDP/Cux transcription factor. Mol Cell. 2004;14(2):207-219.

11. Duncan EM, et al. Cathepsin L proteolytically processes histone $\mathrm{H} 3$ during mouse embryonic stem cell differentiation. Cell. 2008;135(2):284-294.

12. Shih NY, et al. Congenital nephrotic syndrome in mice lacking CD2-associated protein. Science. 1999;286(5438):312-315.

13. Shih NY, Li J, Cotran R, Mundel P, Miner JH, Shaw AS. CD2AP localizes to the slit diaphragm and binds to nephrin via a novel C-terminal domain. Am J Pathol. 2001;159(6):2303-2308.

14. Lehtonen S, Zhao F, Lehtonen E. CD2-associated protein directly interacts with the actin cytoskeleton. Am J Physiol Renal Physiol. 2002;283(4):F734-F743.

15. Kim JM, et al. CD2-associated protein haploinsufficiency is linked to glomerular disease susceptibility. Science. 2003;300(5623):1298-1300.

16. Huber TB, et al. Bigenic mouse models of focal segmental glomerulosclerosis involving pairwise interaction of CD2AP, Fyn, and synaptopodin. J Clin Invest. 2006;116(5):1337-1345.

17. Grunkemeyer JA, Kwoh C, Huber TB, Shaw AS. $\mathrm{CD} 2$-associated protein (CD2AP) expression in podocytes rescues lethality of CD2AP deficiency. J Biol Chem. 2005;280(33):29677-29681.

18. Schiffer M, Mundel P, Shaw AS, Bottinger EP. A novel role for the adaptor molecule CD2-associated
Address correspondence to: Sanja Sever, Division of Nephrology, Massachusetts General Hospital, Harvard Medical School, 149 13th St. 8th Floor, Boston, Massachusetts 02129, USA. Phone: 617.724.8922; Fax: 617.726.5669; E-mail: ssever@partners.org. Or to: Jochen Reiser, Division of Nephrology and Hypertension, Leonard Miller School of Medicine, University of Miami, 1580 NW 10th Ave. Suite 633-A, Miami, Florida 33136, USA. Phone: 305.243.2349; Fax: 305.243.2309; E-mail: jreiser@med.miami.edu. protein in transforming growth factor-beta-induced apoptosis. J Biol Chem. 2004;279(35):37004-37012.

19. Woroniecki RP, Schiffer M, Shaw AS, Kaskel FJ, Bottinger EP. Glomerular expression of transforming growth factor-beta (TGF-beta) isoforms in mice lacking CD2-associated protein. Pediatr Nephrol. 2006;21(3):333-338.

20. Asanuma K, Campbell KN, Kim K, Faul C, Mundel P. Nuclear relocation of the nephrin and CD2AP-binding protein dendrin promotes apoptosis of podocytes. Proc Natl Acad Sci U S A. 2007;104(24):10134-10139.

21. Bottinger EP, Bitzer M. TGF-beta signaling in renal disease. J Am Soc Nephrol. 2002;13(10):2600-2610.

22. Schiffer $M$, et al. Apoptosis in podocytes induced by TGF- $\beta$ and Smad7. J Clin Invest. 2001;108(6):807-816.

23. Ju W, et al. Renal gene and protein expression signatures for prediction of kidney disease progression. Am J Pathol. 2009;174(6):2073-2085.

24. Reiser J, et al. Induction of B7-1 in podocytes is associated with nephrotic syndrome. J Clin Invest. 2004; 113(10):1390-1397.

25. Lohmuller T, et al. Toward computer-based cleavage site prediction of cysteine endopeptidases. Biol Chem. 2003;384(6):899-909.

26. Huber TB, et al. Nephrin and CD2AP associate with phosphoinositide 3-OH kinase and stimulate AKT-dependent signaling. Mol Cell Biol. 2003; 23(14):4917-4928.

27. Clement LC, et al. Podocyte-secreted angiopoietinlike-4 mediates proteinuria in glucocorticoid-sensitive nephrotic syndrome. Nat Med. 2011;17(1):117-122.

28. Zhang SY, et al. c-mip impairs podocyte proximal signaling and induces heavy proteinuria. Sci Signal. 2010;3(147):ra39.

29. Löwik MM, et al. Focal segmental glomerulosclerosis in a patient homozygous for a CD2AP mutation. Kidney Int. 2007;72(10):1198-1203.

30. Gigante M, et al. CD2AP mutations are associated with sporadic nephrotic syndrome and focal segmental glomerulosclerosis (FSGS). Nephrol Dial Transplant. 2009;24(6):1858-1864.

31. Derynck R, Zhang YE. Smad-dependent and Smadindependent pathways in TGF-beta family signaling. Nature. 2003;425(6958):577-584.

32. Xavier S, et al. TbetaRI independently activates Smad- and CD2AP-dependent pathways in podocytes. J Am Soc Nephrol. 2009;20(10):2127-2137.

33. Patrakka J, Tryggvason K. New insights into the role of podocytes in proteinuria. Nat Rev Nephrol. 2009;5(8):463-468.

34. Benzing T. Signaling at the slit diaphragm. J Am Soc Nephrol. 2004;15(6):1382-1391.

35. Jones $\mathrm{N}$, et al. Nck adaptor proteins link nephrin to the actin cytoskeleton of kidney podocytes. Nature. 2006;440(7085):818-823.

36. Verma R, Kovari I, Soofi A, Nihalani D, Patrie K, Holzman LB. Nephrin ectodomain engagement results in Src kinase activation, nephrin phosphorylation, Nck recruitment, and actin polymerization. J Clin Invest. 2006;116(5):1346-1359.

37. Robinson PJ, et al. Oligomerization cycle of dynamin directly regulates actin cytoskeleton. Nat Med.
In press.

38. Liu G, Clement L, Kanwar YS, Avila-Casado C, Chugh SS. ZHX proteins regulate podocyte gene expression during the development of nephrotic syndrome. J Biol Chem. 2006;281(51):39681-39692.

39. Susztak K, Raff AC, Schiffer M, Bottinger EP. Glucose-induced reactive oxygen species cause apoptosis of podocytes and podocyte depletion at the onset of diabetic nephropathy. Diabetes. 2006;55(1):225-233.

40. Asanuma K, et al. Dendrin location in podocytes is associated with disease progression in animal and human glomerulopathy. Am J Nephrol. 2011; 33(6):537-549.

41. Campbell KN, Gupta R, Patrakka J, Shaw AS, Tryggvason K, Mundel PH. Genetic deletion of dendrin delays the onset of proteinuria and improves survival of CD2AP null mice. ASN Renal Week 2010, Abstract F-PO1915.

42. Mundel P, et al. Rearrangements of the cytoskeleton and cell contacts induce process formation during differentiation of conditionally immortalized mouse podocyte cell lines. Exp Cell Res. 1997; 236(1):248-258

43. Dustin ML, et al. A novel adaptor protein orchestrates receptor patterning and cytoskeletal polarity in T-cell contacts. Cell. 1998;94(5):667-677.

44. Mundel P, Heid HW, Mundel TM, Krüger M, Reiser J, Kriz W. Synaptopodin: an actin-associated protein in telencephalic dendrites and renal podocytes. J Cell Biol. 1997;139(1):193-204.

45. Ishidoh K, Kominami E. Multi-step processing of procathepsin L in vitro. FEBS Lett. 1994;352(3):281-284.

46. Kaplan JM, et al. Mutations in ACTN4, encoding alpha-actinin-4, cause familial focal segmental glomerulosclerosis. Nat Genet. 2000;24(3):251-256.

47. Holzman LB, St. John P, Kovari I, Verma R, Holthofer $\mathrm{H}$, Abrahamson D. Nephrin is localized to the slit pore of the glomerular epithelial cell. Kidney Int. 1999;56(4):1481-1491.

48. Kopp JB, et al. Transgenic mice with increased plasma levels of TGF-beta 1 develop progressive renal disease. Lab Invest. 1996;74(6):991-1003.

49. Xiao Z. Identification and Characterization of Novel Glomerulus-Associated Genes and Proteins [dissertation]. Stockholm, Sweden: Karolinska Institutet; 2010.

50. Reiser J, et al. Podocyte migration during nephrotic syndrome requires a coordinated interplay between cathepsin L and $3 \alpha$ integrin. J Biol Chem. 2004; 279(33):34827-34832

51 . Kim SW, et al. Increased expression and apical targeting of renal $\mathrm{ENaC}$ subunits in puromycin aminonucleoside-induced nephrotic syndrome in rats. Am J Physiol Renal Physiol. 2004;286(5):F922-F935.

52. Wei C, et al. Modification of kidney barrier function by the urokinase receptor. Nat Med. 2008;14(1):55-63

53. Ju W, et al. Deletion of Smad2 in mouse liver reveals novel functions in hepatocyte growth and differentiation. Mol Cell Biol. 2006;26(2):654-667.

54. Damke H, Baba T, Warnock DE, Schmid SL. Induction of mutant dynamin specifically blocks endocytic coated vesicle formation. J Cell Biol. 1994; 127(4):915-934. 